\title{
GRAPHS CONTAINING EVERY 2-FACTOR
}

\author{
ALEXANDR V. KOSTOCHKA ${ }^{\dagger}$ AND GEXIN YU
}

\begin{abstract}
For a graph $G$, let $\sigma_{2}(G)=\min \{d(u)+d(v): u v \notin E(G)\}$. We prove that every $n$-vertex graph $G$ with $\sigma_{2}(G) \geq 4 n / 3-1$ contains each 2-regular $n$-vertex graph. This extends a theorem due to Aigner and Brandt and to Alon and Fisher.
\end{abstract}

\section{INTRODUCTION}

One of the basic results on Hamiltonian cycles in graphs, Dirac's theorem [8], says that every $n$-vertex graph $G$ with minimum degree, $\delta(G)$, at least $n / 2$ contains a Hamiltonian cycle. The value $n / 2$ is best possible. Furthermore, the condition $\delta(G) \geq n / 2$ does not guarantee that $G$ contains each 2-factor. For example, if $n=3 k$, then the complete 3-partite graph $K_{k-1, k, k+1}$ has minimum degree $2 k-1=\frac{2 n}{3}-1$ and contains no triangle factor. Corrádi and Hajnal [6] proved that a $3 k$-vertex graph $G$ with $\delta(G) \geq 2 k$ contains $k$ disjoint triangles. The condition $\delta(G) \geq 2 k$ cannot be weakened. Aigner and Brandt [1], and independently Alon and Fisher [2] (for $n$ sufficiently large) extended the Corrádi-Hajnal Theorem as follows.

Theorem 1. If $G$ is an $n$-vertex graph with $\delta(G) \geq(2 n-1) / 3$, then $G$ contains each $n$-vertex graph $H$ with $\Delta(H) \leq 2$.

The square, $P_{n}^{2}$, of an $n$-vertex path $P_{n}$ is an $n$-vertex graph $G$ whose vertices can be ordered $v_{1}, v_{2}, \ldots, v_{n}$ so that $v_{i} v_{j} \in E(G)$ if and only if $1 \leq|i-j| \leq 2$. It is easy to check that $P_{n}^{2}$ contains every $n$-vertex graph with maximum degree 2. Fan and Kierstead [10] proved the following strengthening of Theorem 1.

Theorem 2. Every n-vertex graph $G$ with $\delta(G) \geq(2 n-1) / 3$ contains $P_{n}^{2}$.

Ore [16] gave a different sufficient condition for hamiltonicity: he proved that every $n$ vertex graph $G$ with

$$
\sigma_{2}(G)=\min _{x y \notin E(G)}\{\operatorname{deg}(x)+\operatorname{deg}(y)\} \geq n
$$

contains a Hamiltonian cycle. Justesen [11] proved an Ore- type version of the CorrádiHajnal Theorem by showing that every $n$-vertex graph $G$ with $\sigma_{2}(G) \geq 4 n / 3$ contains $\lfloor n / 3\rfloor$

Date: June 7, 2011.

1991 Mathematics Subject Classification. 05C35, 05C70.

Key words and phrases. graph packing, Ore-type degree conditions, 2-factors.

$\dagger$ Research supported in part by NSF grants DMS-0650784 and DMS-0965587 and by RFBR grant 08-0100673.

$\ddagger$ Research supported in part by NSF grant DMS-0852452. 
disjoint triangles. Enomoto [9], and Wang [18] sharpened this result. In particular, they proved the following.

Theorem 3. For each positive integer $k$, every $3 k$-vertex graph $G$ with $\sigma_{2}(G) \geq 4 k-1$ contains $k$ disjoint triangles.

In [15], Theorem 3 was extended as follows.

Theorem 4. Each n-vertex graph $G$ with $\sigma_{2}(G) \geq \frac{4 n}{3}-1$ contains all spanning subgraphs whose components are isomorphic to graphs in $\mathcal{H}=\left\{K_{1}, K_{2}, C_{3}, K_{4}^{-}, C_{5}^{+}\right\}$.

Here $K_{4}^{-}$denotes the graph obtained from the complete 4 -vertex graph $K_{4}$ by deleting an edge, and $C_{5}^{+}$is the graph obtained from the 5 -cycle $C_{5}$ by adding an edge.

The aim of this paper is to prove the following Ore-type analogue of Theorem 1.

Theorem 5. Each n-vertex graph $G$ with

$$
\sigma_{2}(G) \geq \frac{4 n}{3}-1
$$

contains every n-vertex graph $H$ with $\Delta(H) \leq 2$.

This theorem is also a step toward an Ore-type analogue of a conjecture by Bollobás and Eldridge [3], and Catlin [5] on packing of graphs (se Conjecture 2 below).

Two $n$-vertex graphs $G_{1}$ and $G_{2}$ pack if there exist injective mappings of their vertex sets onto $[n]$ such that the images of the edge sets do not intersect. Equivalently, $G_{1}$ and $G_{2}$ pack if $G_{1}$ is isomorphic to a subgraph of the complement of $G_{2}$. This concept leads to a natural generalization of a number of problems in extremal graph theory, such as the existence of a fixed subgraph, equitable colorings, and Turán-type problems. In the language of packing, some embedding problems may sound more natural. For example, let $\theta(G)=$ $\max \{d(u)+d(v): u v \in E(G)\}$. Then in the language of packings, the above-mentioned Ore's theorem [16] says that every $n$-vertex graph $G$ with $\theta(G) \leq n-2$ packs with the $n$-cycle $C_{n}$, and our Theorem 5 says that each n-vertex graph $G$ with $\theta(G) \leq \frac{2 n}{3}-1$ packs with every $n$ vertex graph $H$ such that $\Delta(H) \leq 2$. Note that while $\sigma_{2}$ relates to non-adjacent vertices, $\theta(G)$ is a characteristic of edges in $G$. In [12], this parameter is called the maximum Ore-degree of $G$.

The study of extremal graph packing problems started in the 1970s by Bollobás and Eldridge [3], Sauer and Spencer [17], and Catlin [4]. They considered graph packing under degree constrains. In particular, Bollobás and Eldridge [3], and Catlin [5] stated the following BEC-conjecture:

Conjecture 1. If $G_{1}$ and $G_{2}$ are $n$-vertex graphs and $\left(\Delta\left(G_{1}\right)+1\right)\left(\Delta\left(G_{2}\right)+1\right) \leq n+1$, then $G_{1}$ and $G_{2}$ pack.

This is sharp, if true. Theorem 1 above is the case $\Delta\left(G_{2}\right)=2$ of the BEC-conjecture. Csaba, Shokoufandeh, and Szemerédi [7] also proved the conjecture in the case $\Delta\left(G_{2}\right) \leq 3$ and $n$ is huge, but otherwise, the BEC-conjecture is wide open. 
The following Ore-type analogue of the BEC-conjecture was posed in [13].

Conjecture 2. If $G_{1}$ and $G_{2}$ are $n$-vertex graphs and $\left(0.5 \theta\left(G_{1}\right)+1\right)\left(\Delta\left(G_{2}\right)+1\right) \leq n+1$, then $G_{1}$ and $G_{2}$ pack.

Thus Theorem 5 verifies the case $\Delta\left(G_{2}\right)=2$ of Conjecture 2 . In fact, we will prove the slightly stronger result than Theorem 5 , which in the language of packing is as follows.

Theorem 6. Each n-vertex graph $G$ such that

$$
\theta(G) \leq \frac{2 n}{3}-1
$$

packs with every $n$-vertex graph $H$ such that $\theta(H) \leq 4$.

In Section 2 we outline the proof (it will have 6 stages) and give some definitions. In Section 3, we state several lemmas that are our main tools for embedding a sequence of graphs into $G$ such that the last graph in the sequence is the desired one. In the same section we also prove two of the lemmas that have shorter proofs. The longer proofs are postponed to the last three sections. In Section 4 we show how Stages 2-4 work, and in Section 5 how Stages 5 and 6 work. In the last three sections, we present the proofs for the lemmas from Section 3 .

\section{Outline of the PROOF}

In this section, we introduce useful notions, and describe the idea of the main proof. We use and modify the ideas of Aigner and Brandt [1].

Every component of an $n$-vertex graph $H$ with $\theta(H) \leq 4$, is either a path, or a cycle, or a $K_{1,3}$. We will show the following statement which is slightly stronger than Theorem 6:every $n$-vertex graph $G$ satisfying (1), contains each $n$-vertex graph $H$ whose components are in $\mathcal{F}=\left\{K_{1}, K_{2}, K_{4}^{-}\right\} \cup\left\{C_{l_{j}}: 3 \leq l_{j} \leq n\right\}$.

A double $i$-lasso (further, simply an $i$-lasso), $D_{i}$, consists of a path $x_{1}, x_{2}, \cdots, x_{i}$ with the additional edges $x_{1} x_{3}$ and $x_{i-2} x_{i}$. For example, $D_{4}=K_{4}^{-}$.

The strategy of our proof is as follows. Let an $n$-vertex graph $G$ satisfy (1) and let $H$ be an $n$-vertex graph whose components are in $\mathcal{F}$. We will first embed into $G$ an auxiliary graph $H_{1}$ each of whose components has at most 5 vertices, and belongs to $\left\{K_{1}, K_{2}, K_{3}, K_{4}^{-}, C_{5}^{+}\right\}$. Then using this embedding and (1), we will step by step find embeddings of graphs that are closer and closer to $H$. The first main goal will be to construct embedding into $G$ of a graph whose components are double lassoes which have the same orders as the corresponding cycle components of $H$. Based on an embedding of this graph and Property (1), we will be able to embed $H$ into $G$. We do this in several stages.

Stage 1. First, for each component $R_{j}$ of $H$ that is a cycle of length $\ell_{j}$, we represent $\ell_{j}$ as the sum of small summands according to the following rules.

(A) If $\ell_{j} \equiv 0(\bmod 6)$, then $\ell_{j}=6+\cdots+6$.

(B) If $\ell_{j} \equiv 3(\bmod 6)$ and $\ell_{j} \geq 9$, then $\ell_{j}=6+\cdots+6+3$. 
(C) If $\ell_{j} \equiv 1(\bmod 6)$, then $\ell_{j}=6+\cdots+6+3+4$.

(D) If $\ell_{j} \equiv 2(\bmod 6)$, then $\ell_{j}=6+\cdots+6+4+4$.

(E) If $\ell_{j} \equiv 4(\bmod 6)$ and $\ell_{j} \geq 10$, then $\ell_{j}=6+\cdots+6+4$.

(F) If $\ell_{j} \equiv 5(\bmod 6)$ and $\ell_{j} \geq 11$, then $\ell_{j}=6+\cdots+6+4+4+3$.

(G) If $\ell_{j} \leq 5$, then $\ell_{j}=\ell_{j}$.

Let $H_{1}$ be obtained from $H$ by replacing each $C_{5}$-component $R_{j}$ of $H$ with $C_{5}^{+}$and replacing for each $\ell_{j} \geq 6$, the component that is the cycle $C_{\ell_{j}}$ with a set $M_{j}$ of disjoint $K_{3}$-components and $K_{4}^{-}$-components so that each summand 4 in the above representation of $\ell_{j}$ corresponds to a $K_{4}^{-}$-component, each summand 3 corresponds to a $K_{3}$, and each summand 6 corresponds to two disjoint $K_{3}$ s. By construction, $H_{1}$ is an $n$-vertex graph each of whose component is in $\mathcal{F}_{1}=\left\{K_{1}, K_{2}, K_{4}^{-}, K_{3}, C_{5}^{+}\right\}$. By Theorem $4, G$ contains a copy of $H_{1}$. We call the graph $H_{1}$ an initial $H$-approximation. (Calling a graph $H^{\prime}$ an $H$-approximation we mean that $H^{\prime}$ in a sense does not much differ from $H$, and will embed into $G H$-approximations that are closer and closer to $H$.)

Stage 2. Starting from an embedding into $G$ of the $H$-approximation $H^{\prime}=H_{1}$ with given sets $M_{j}$, we will embed into $G$ an $H$-approximation $H_{2}$ such that every summand 6 in $\ell_{j}$ corresponds to a 6-lasso. We obtain this in steps: Each step starts from an $H$-approximation $H^{\prime}$ embedded into $G$ and finishes with an embedding into $G$ of a graph $H^{\prime \prime}$ that is obtained from $H^{\prime}$ by replacing two $K_{3}$-components from the same $M_{j}$ in $H^{\prime}$ with one 6 -lasso. We will say that $H^{\prime \prime}$ is an $H$-approximation $H^{\prime \prime}$ slightly better than $H^{\prime}$. As before, the orders of the components in each $M_{j}$ sum to $\ell_{j}$.

Stage 3. Starting from the $H$-approximation $H^{\prime}=H_{2}$ with given sets $M_{j}$, we will embed into $G$ an $H$-approximation $H_{3}$ such that each summand 3 in the representation of an $\ell_{j} \geq 9$ corresponds to a part of a 9-lasso in $\mathrm{H}_{2}$. Again, we do it in steps: Each step starts from an $H$-approximation $H^{\prime}$ embedded into $G$ and finishes with an embedding into $G$ of a graph $H^{\prime \prime}$ that is obtained from $H^{\prime}$ by replacing a $K_{3}$-component and a 6-lasso from the same $M_{j}$ in $H^{\prime}$ with one 9-lasso.

Stage 4. Starting from the $H$-approximation $H^{\prime}=H_{3}$ with given sets $M_{j}$, we will embed into $G$ an $H$-approximation $H_{4}$ such that the summand 4 and the summand 3 (if both exist in $\ell_{j}$ ) correspond to a 7-lasso. Here, in each step, we embed into $G$ the graph $H^{\prime \prime}$ obtained from the starting graph $H^{\prime}$ by replacing a $K_{3}$-component and a $K_{4}^{-}$-component from the same $M_{j}$ with a 7 -lasso. Note that this stage is needed only if $\ell_{j}=7$.

Stage 5. Starting from the $H$-approximation $H^{\prime}=H_{4}$ with given sets $M_{j}$, we will embed $G$ an $H$-approximation $H_{5}$ such that each cycle $C_{j}$ in $H$ with $\ell_{j} \geq 6$ corresponds to an $\ell_{j}$ lasso in $H_{5}$. We start from $H^{\prime}=H_{4}$ by replacing each $C_{5}^{+}$-component with a $C_{5}$-component. Since $H^{\prime} \subseteq H_{4}$, we have an embedding of $H^{\prime}$ into $G$. In each step of the stage, given an embedding into $G$ of an $H$-approximation $H^{\prime}$, we find an embedding into $G$ of a graph $H^{\prime \prime}$ that is obtained from $H^{\prime}$ by replacing two disjoint lassoes (say of orders $z_{1}$ and $z_{2}$ ) from the same $M_{j}$ with a $\left(z_{1}+z_{2}\right)$-lasso. Recall that we view $K_{4}^{-}$as a 4 -lasso. 
Stage 6. We start from the $H$-approximation $H^{\prime}=H_{5}$ and in each step from an embedding of $H^{\prime}$ into $G$ we obtain an embedding into $G$ of a graph $H^{\prime \prime}$ that is obtained from $H^{\prime}$ by replacing an $\ell_{j}$-lasso for some $\ell_{j} \geq 6$ with an $\ell_{j}$-cycle. By construction, the last $H$ approximation embedded into $G$ will coincide with $H$.

Note that if $\ell_{j} \geq 6$, then before finding an embedding of $H$ into $G$ we had an embedding into $G$ of an $H^{\prime}$ which is obtained from $H$ by replacing $C_{j}$ with an $\ell_{j}$-lasso. So, practically repeating our proof of Theorem 5 one can derive the following slightly stronger result.

Theorem 7. Each n-vertex graph $G$ satisfying (1) contains every n-vertex graph $H$ such that every component of $H$ is either a cycle, or $K_{1}$, or $K_{2}$, or a double lasso $D_{\ell}$ for $\ell \neq 5$.

\section{BASIC LEMMAS}

For two subgraphs $X$ and $X^{\prime}$ of a graph $F$, let $E_{F}\left(X, X^{\prime}\right)$ denote the set of edges connecting $X$ with $X^{\prime}$ in $F$ and $e_{F}\left(X, X^{\prime}\right)=\left|E_{F}\left(X, X^{\prime}\right)\right|$. For $X \subset V(F)$ and $v \in V(F)$, let $d_{F}(v, X)=$ $e_{F}(\{v\}, X)$. If the graph $F$ is clear from the context, we will drop the subscript.

In Stages 5 and 6 , an $n$-vertex graph $H^{\prime \prime}$ is an $H$-quasi-approximation, if there exists an $H$-approximation $H^{\prime}$ such that $H^{\prime \prime}$ is obtained from $H^{\prime}$ by replacing a $C_{5}$-component with a $D_{5}$-component. In this case, $H^{\prime}$ is slightly better than $H^{\prime \prime}$. A weak $H$-approximation is either an $H$-approximation or $H$-quasi-approximation.

From now on, $G$ is an $n$-vertex graph satisfying (1) with a fixed embedding $\Psi$ of a weak $H$-approximation $H^{\prime}$. When speaking of vertices and subgraphs of $H^{\prime}$, we usually will mean $H^{\prime}$ as the subgraph of $G$ defined by $\Psi$. By definition, in Stages $2-4$, the notions of an $H$-approximation and a weak $H$-approximation coincide.

Given a pair $\left(G, H^{\prime}\right)$ a gadget is a 4-element vertex set $Y=Y_{1} \cup Y_{2}$ of $H^{\prime}$, where the 2-element sets $Y_{1}$ and $Y_{2}$ are chosen as follows. If $H^{\prime}$ is an $H$-quasi-approximation, then $Y_{1}$ consists of the first two vertices and $Y_{2}$ consists of the last two vertices of the only $D_{5^{-}}$ component in $H^{\prime}$. If $H^{\prime}$ is an $H$-approximation, then each set $Y_{i}$ is formed either by the two first (or the two last) vertices of a double lasso in $H^{\prime}$, or by the two nonadjacent vertices in a $K_{4}^{-}$-component in $H^{\prime}$. The component of $H^{\prime}$ containing $Y_{i}$ will be called the $Y_{i}$-block, $i=1,2$. It may happen that the $Y_{1}$-block and the $Y_{2}$-block coincide. In this case, $Y_{1}$ and $Y_{2}$ contain the ends of the same double lasso in $H^{\prime}$. By default, we will assume that $Y_{1}=\left\{y_{1}, y_{1}^{\prime}\right\}$ and $Y_{2}=\left\{y_{2}, y_{2}^{\prime}\right\}$.

For a gadget $Y$, a $Y$-connector $Y^{\prime}$ is a 4-element vertex set obtained from $Y$ either (i) by deleting some $y \in Y_{i}$ (where $i \in\{1,2\}$ ) and adding some $y_{0}$ adjacent to $Y_{i}-y$ and $Y_{3-i}$, or (ii) by deleting some $y \in Y_{1}$ and $y^{\prime} \in Y_{2}$ and adding $z$ and $z^{\prime}$ such that $z$ is adjacent to $Y_{1}-y$ and $z^{\prime}$, and $z^{\prime}$ is also adjacent to $Y_{2}-y^{\prime}$. The idea of a $Y$-connector is the following. If $Y_{1}$ and $Y_{2}$ are formed by the first two and the last two vertices in the same double-lasso-component of $H^{\prime}$ with vertex set $D$, then for each $Y$-connector $Y^{\prime}$, the graph $G\left[(D-Y) \cup Y^{\prime}\right]$ contains a cycle with $|D|$ vertices. If the $Y_{1}$-block with vertex set $V_{j}$ and the $Y_{2}$-block with vertex set 
$V_{j^{\prime}}$ are distinct, then for each $Y$-connector $Y^{\prime}$, the graph $G\left[\left(V_{j} \cup V_{j^{\prime}}-Y\right) \cup Y^{\prime}\right]$ contains a double lasso with $\left|V_{j}\right|+\left|V_{j^{\prime}}\right|$ vertices.

For a path $P=\left(u_{1}, \ldots, u_{k}\right)$ and a set $Y \subset V(G)$ and for $2 \leq i \leq k-1$, let $d_{3}\left(u_{i}, Y\right)=$ $e_{G}\left(\left\{u_{i-1}, u_{i}, u_{i+1}\right\}, Y\right)$. The next lemma elaborates Proposition 3 in [1].

Lemma 1. Let $H^{\prime}$ be a weak $H$-approximation embedded into $G$. Let $Y=Y_{1} \cup Y_{2}$ be a gadget. Let $P=\left(u_{1}, \ldots, u_{\ell+1}\right)$ be a path of length $\ell$ in $H^{\prime}$ disjoint from $Y$. Let $U=\left\{u_{1}, \ldots, u_{\ell+1}\right\}$. Assume that the set $Y \cup U$ does not contain a $Y$-connector $Y^{\prime}$ such that $G\left[Y \cup U-Y^{\prime}\right]$ contains a path of length $\ell$ from $u_{1}$ to $u_{l+1}$.

(c1) If $u_{i} \in U$ is adjacent to $y \in Y_{1}$ and $y^{\prime} \in Y_{2}$, then a vertex $y^{\prime \prime} \in Y-\left\{y, y^{\prime}\right\}$ cannot be adjacent (in $G$ ) to all of the neighbors of $u_{i}$ in $P$.

(c2) If $d_{3}\left(u_{i}, Y\right) \geq 9$, then $d\left(u_{i}, Y\right) \leq 2$. Furthermore, if $d_{3}\left(u_{i}, Y\right) \geq 9$ and $d\left(u_{i}, Y\right)=2$, then $u_{i}$ cannot have neighbors both in $Y_{1}$ and $Y_{2}$.

(c3) If some $u_{i}$ satisfies $d_{3}\left(u_{i}, Y\right) \geq 9$, then the possible degree sequences of $\left(u_{i-1}, u_{i}, u_{i+1}\right)$ in $Y$ are $(4,2,3),(3,2,4),(4,2,4)$ and $(4,1,4)$.

(c4) If $d_{3}\left(u_{i}, Y\right)+d_{3}\left(u_{i+1}, Y\right) \geq 17$ for some $i$ and $d_{3}\left(u_{i}, Y\right) \geq 9$, then the possible degree sequences in $Y$ for $\left(u_{i-1}, u_{i}, u_{i+1}, u_{i+2}\right)$ are

$$
(4,2,3,3),(3,2,4,2),(4,1,4,3),(4,2,4,2),(4,2,4,1) \text {. }
$$

Furthermore, the subgraph of $G$ induced by $Y \cup\left\{u_{i-1}, u_{i}, u_{i+1}, u_{i+2}\right\}$ is one of the graphs in Figure 1 (up to isomorphism).

Proof of Lemma 1. Statement (c1) is clear, since otherwise $G\left[U-u_{i}+y^{\prime \prime}\right]$ contains an $\ell$-path, and $Y-y^{\prime \prime}+u_{i}$ is a $Y$-connector.

To show $(\mathrm{c} 2)$, observe that if $d\left(u_{i-1}, Y\right)+d\left(u_{i+1}, Y\right) \geq 5$, then $u_{i-1}$ and $u_{i+1}$ have a common neighbor in $Y$, and if $d\left(u_{i-1}, Y\right)+d\left(u_{i+1}, Y\right) \geq 6$, then $u_{i-1}$ and $u_{i+1}$ have at least two common neighbors in $Y$. So, if $d\left(u_{i}, Y\right) \geq 3$, then we can always find $y \in Y_{1}$ and $y^{\prime} \in Y_{2}$ such that $u_{i}$ is adjacent to $y$ and $y^{\prime}$, and $y^{\prime \prime} \in Y-\left\{y, y^{\prime}\right\}$ is a common neighbor of $u_{i-1}$ and $u_{i+1}$, a contradiction to $(\mathrm{c} 1)$. Furthermore, if $d\left(u_{i}, Y\right)=2$ and $u_{i}$ has neighbors in both $Y_{1}$ and $Y_{2}$, then the same argument works.

By (c2), (c3) is clear.

Now we prove $(\mathrm{c} 4)$. By $(\mathrm{c} 3),\left(u_{i-1}, u_{i}, u_{i+1}\right)$ has one of the four possible degree sequences in $Y$. In all these sequences, $d\left(u_{i+1}, Y\right) \geq 3$ and hence $d_{3}\left(u_{i+1}, Y\right) \leq 8$ by (c2). On the other hand, $d_{3}\left(u_{i+1}, Y\right) \geq 17-d_{3}\left(u_{i}, Y\right) \geq 7$.

Suppose first that $u_{i}$ and $u_{i+2}$ have a common neighbor $y \in Y$. We may assume that $y \in Y_{1}$. Since $U-u_{i+1}+y$ contains a path of length $\ell$ and by $(\mathrm{c} 3) d\left(u_{i+1}, Y\right) \geq 3, u_{i+1}$ cannot be adjacent to the vertex in $Y_{1}-y$. It follows that $d\left(u_{i+1}, Y\right)=3$. To have $d_{3}\left(u_{i}, Y\right) \geq 9$, by (c2), we need $d\left(u_{i}, Y\right)=2$ and $d\left(u_{i-1}, Y\right)=4$. So, we have Case (A) in Figure 1.

Suppose now that $u_{i}$ and $u_{i+2}$ have no common neighbor $y \in Y$. By (c2) and $d_{3}\left(u_{i}, Y\right) \geq 9$, for this we need $d\left(u_{i+1}, Y\right)=4$. If $d\left(u_{i}, Y\right)=1$, then to have $d_{3}\left(u_{i}, Y\right)+d_{3}\left(u_{i+1}, Y\right) \geq 17$, we need $d\left(u_{i-1}, Y\right)=4$ and $d\left(u_{i+2}, Y\right)=3$. This is Case (D) in Figure 1. So, let $d\left(u_{i}, Y\right)=2$. 
(A)

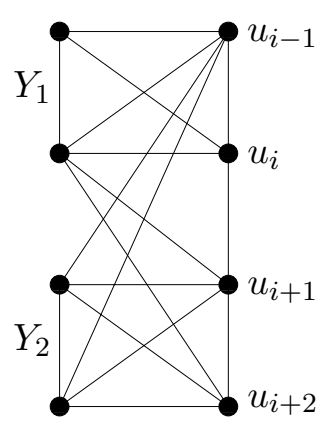

$(4,2,3,3)$

(D)

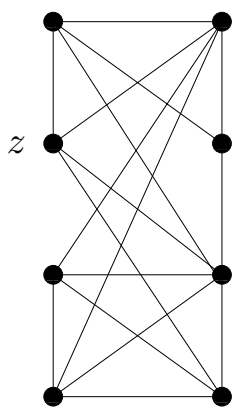

$(4,1,4,3)$
(B)

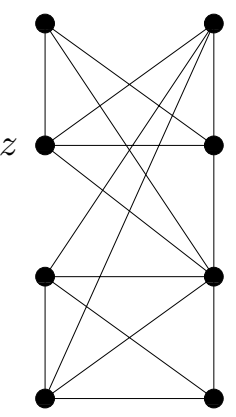

$(3,2,4,2)$

(E)

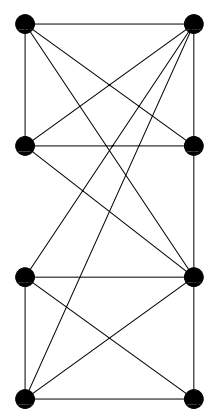

$(4,2,4,2)$
(C)

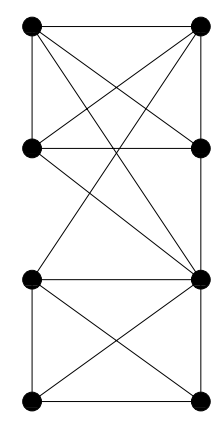

$(3,2,4,2)$

(F)

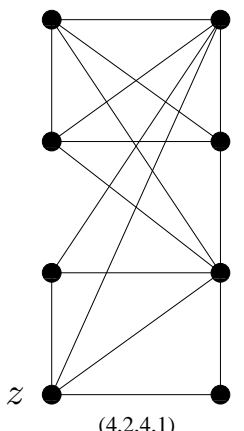

Figure 1

Then by (c2), both neighbors of $u_{i}$ in $Y$ are in the same $Y_{j}$, say, in $Y_{1}$. If $d\left(u_{i+2}, Y\right)=1$, then $d\left(u_{i-1}, Y\right)=4$ and we have Case (F) in Figure 1. Finally, if $d\left(u_{i+2}, Y\right)=2$, then since $u_{i}$ and $u_{i+2}$ have no common neighbor $y \in Y$, both neighbors of $u_{i+2}$ in $Y$ are in $Y_{2}$ and we have one of Cases (B), (C), or (E) in Figure 1.

Lemma 2. Let $H^{\prime}$ be a weak $H$-approximation $H^{\prime}$ in Stage 5 or 6 . Let $Y=Y_{1} \cup Y_{2}$ be a gadget and $F \subset V\left(H^{\prime}\right)$ be such that $H^{\prime}[F]=C_{k}$ with $k \geq 5$ is a component of $H^{\prime}$ disjoint from $Y$. Let $e_{G}(Y, F)>8 k / 3$.

(a) If $k \geq 6$, then there exists a $Y$-connector $Y^{\prime} \subset Y \cup F$ such that $G\left[(Y \cup F)-Y^{\prime}\right]$ contains $a C_{k}$.

(b) If $k=5$, then there exists a $Y$-connector $Y^{\prime} \subset Y \cup F$ such that $G\left[(Y \cup F)-Y^{\prime}\right]$ either contains a $C_{5}$ or contains the double lasso $D_{5}$.

(c) Moreover, if $k=5$, and $H^{\prime}$ is an $H$-quasi-approximation (and, by definition, $Y_{1}$ and $Y_{2}$ belong to the only $D_{5}$-component of $\left.H^{\prime}\right)$, then $G\left[F \cup F_{1}\right]$ contains two disjoint 5-cycles.

Proof. Let $H^{\prime}[F]=C_{k}=\left(u_{1}, \ldots, u_{k}\right)$. Recall that $d_{3}\left(u_{i}, Y\right)=d\left(u_{i-1}, Y\right)+d\left(u_{i}, Y\right)+$ $d\left(u_{i+1}, Y\right)$. Since $e_{G}(F, Y)>8 k / 3$, there exists $i$ such that $d_{3}\left(u_{i}, Y\right)+d_{3}\left(u_{i+1}, Y\right) \geq 17$. By 
flipping the order of vertices in $F$ if needed, we may assume that $d_{3}\left(u_{i}, Y\right) \geq 9$. Lemma 1 yields that the possible degree sequences towards $Y$ of $\left(u_{i-1}, u_{i}, u_{i+1}, u_{i+2}\right)$ are

$$
(4,2,3,3),(3,2,4,2),(4,1,4,3),(4,2,4,2),(4,2,4,1)
$$

and that $G\left[Y \cup\left\{u_{i-1}, u_{i}, u_{i+1}, u_{i+2}\right\}\right]$ is one of the graphs in Figure 1 (up to isomorphism).

Recall that by default, $Y_{1}=\left\{y_{1}, y_{1}^{\prime}\right\}$ and $Y_{2}=\left\{y_{2}, y_{2}^{\prime}\right\}$. Assume that $d_{i}=d\left(y_{i}, F\right) \geq$ $d\left(y_{i}^{\prime}, F\right)=d_{i}^{\prime}$ for $i=1,2$.

Proof of (a): Suppose $k \geq 6$. Consider configurations (A), (C), and (E) in Figure 1. In each of them for any choice of $y_{1} \in Y_{1}$ and $y_{2} \in Y_{2}$, there is a path from $y_{1}^{\prime}$ to $y_{2}^{\prime}$ via $u_{i}$ and $u_{i+1}$. This path will be our $Y$-connector. Furthermore, the graph $G\left[F \cup\left\{y_{1}, y_{2}\right\}-\left\{u_{i}, u_{i+1}\right\}\right]$ contains a Hamiltonian path from $y_{1}$ to $y_{2}$, say $\left(y_{1}=z_{1}, z_{2}, \ldots, z_{k}=y_{2}\right)$. Observe that $e_{G}\left(\left\{y_{1}, y_{2}\right\}, F-\left\{u_{i}, u_{i+1}\right\}\right) \geq d_{1}+d_{2}-3>\frac{4}{3} k-3 \geq k-1$. So, as in the proof of Ore's Theorem, there exists a $j$ such that $y_{1} z_{j+1}, y_{2} z_{j} \in E(G)$. Then $G\left[F \cup\left\{y_{1}, y_{2}\right\}-\left\{u_{i}, u_{i+1}\right\}\right]$ has Hamiltonian cycle $\left(z_{1}, z_{2}, \ldots, z_{j}, z_{k}, z_{k-1}, \ldots, z_{j+1}\right)$.

Consider now configurations (B), (D), and (F). If $y_{1}=z$ (or $\left.y_{2}=z\right)$ in Figure 1, then we obtain a $Y$-connector and $C_{k}$ as above. Thus we may assume that $z=y_{1}^{\prime}$ (or $z=y_{2}^{\prime}$ ) and $d_{1}>d_{1}^{\prime}\left(\right.$ or $\left.d_{2}>d_{2}^{\prime}\right)$. Next, note that if in configuration (B), $y_{2}$ were adjacent to $u_{i+3}$, then the above argument with $\left\{u_{i+1}, u_{i+2}\right\}$ in place of $\left\{u_{i}, u_{i+1}\right\}$ would yield a a proof again. So we may assume that $y_{2} u_{i+3} \notin E(G)$ and thus that $d_{2} \leq k-2$ in configuration (B).

We now estimate $d_{1}=e_{G}(Y, F)-d_{1}^{\prime}-d_{2}-d_{2}^{\prime}$ in (B), (D) and (F). If we replace $\left\{y_{1}, y_{2}\right\}$ by $\left\{y_{1}^{\prime}, y_{2}^{\prime}\right\}$ in the previous argument, then to have $G\left[F \cup\left\{y_{1}^{\prime}, y_{2}^{\prime}\right\}-\left\{u_{i}, u_{i+1}\right\}\right]$ non-Hamiltonian, by Ore's theorem we will have

$d_{1}^{\prime}+d_{2}^{\prime} \leq k-1+3$ and $d_{2} \leq k-2$ in (B) and (F), $d_{1}^{\prime}+d_{2}^{\prime} \leq k-1+2$ and $d_{1} \leq k-1$ in (D).

Set $(i, j)$ equal to $(1,2)$ in $(\mathrm{B})$ and $(\mathrm{F})$ and equal to $(2,1)$ in $(\mathrm{D})$. Then

$$
d_{i}>\left(\frac{8}{3}-2\right) k=\frac{2}{3} k \geq \frac{1}{2} k+1
$$

Now, $Y \cup\left\{u_{i}, u_{i+1}\right\}-\left\{y_{i}, y_{j}^{\prime}\right\}$ contains a Hamiltonian path from $y_{i}^{\prime}$ to $y_{j}$ and so $G[F \cup$ $\left.\left\{y_{j}^{\prime}\right\}-\left\{u_{i}, u_{i+1}\right\}\right]$ is Hamiltonian. It follows from (3) that $y_{i}$ is adjacent to two consecutive vertices of the path $F-\left\{u_{i}, u_{i+1}\right\}$. So, $C_{k} \subseteq G\left[F \cup\left\{y_{i}, y_{j}^{\prime}\right\}-\left\{u_{i}, u_{i+1}\right\}\right]$, as desired.

Proof of $(b)$ : Let $k=5$. Since $e_{G}\left(Y, C_{5}\right) \geq 14$, we have $d\left(u_{i-2}, Y\right) \geq 2$ in graphs (A), (D) and $(\mathrm{E})$, and $d\left(u_{i-2}, Y\right) \geq 3$ in graphs $(\mathrm{B}),(\mathrm{C})$ and $(\mathrm{F})$. We see that in all cases, except (E), the assertion (c1) of Lemma 1 is violated for some subpath of length 3 in our 5-cycle.

In the remaining Case (E), the sequence of degrees in $Y$ for $\left(u_{i-2}, u_{i-1}, u_{i}, u_{i+1}, u_{i+2}\right)$ is $(2,4,2,4,2)$, and the configuration is as in Figure 2.

Thus we may partition $G[Y \cup F]$ into a path from $Y_{1}$ to $Y_{2}$ (non-filled circles in Figure 2) and the lasso $D_{5}$ (filled circles in Figure 2).

Proof of (c): Suppose $H^{\prime}$ is an $H$-quasi-approximation (which means that the four vertices of $Y$ are the vertices of degree 2 in a $D_{5}$-component $F_{1}$ of $H^{\prime}$ ) and $k=5$. Let the fifth vertex of $F_{1}$ (adjacent to all vertices in $Y$ ) be $z$. By the proof of Case 2, it is enough to 


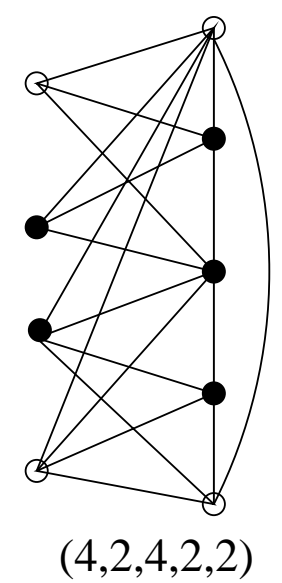

Figure 2. The case $(4,2,4,2,2)$

consider the sequence of degrees toward $Y$ for $\left(u_{i-2}, u_{i-1}, u_{i}, u_{i+1}, u_{i+2}\right)$ equal to $(2,4,2,4,2)$ and the configuration depicted in Figure 2. If $u_{i-2}$ has a neighbor, say, $y_{1}$ in $Y_{1}$, then both $G\left[V\left(F_{1}\right)-y_{1}+u_{i-1}\right]$ and $G\left[F+y_{1}-u_{i-1}\right]$ contain 5-cycles. Otherwise, $N\left(u_{i-2}, Y\right)=Y_{2}$. Then both $G\left[\left\{y_{1}, y_{1}^{\prime}, z, u_{i-1}, u_{i}\right\}\right]$ and $G\left[\left\{y_{2}, y_{2}^{\prime}, u_{i+1}, u_{i+2}, u_{i-2}\right\}\right]$ contain 5-cycles.

The proofs of the next three lemmas will be given in the last three sections.

Lemma 3. Let $H^{\prime}$ be a weak $H$-approximation in Stage 5 or 6 . Let $Y=Y_{1} \cup Y_{2}$ be a gadget and $F=D_{k}$ with $k \geq 6$ be a component of $H^{\prime}$ disjoint from $Y$. If $e_{G}(Y, F)>8 k / 3$, then there exists a $Y$-connector $Y^{\prime} \subset Y \cup F$ such that $G\left[(Y \cup F)-Y^{\prime}\right]$ contains a $D_{k}$.

Lemma 4. Let $H^{\prime}$ be a weak $H$-approximation in Stage 5 or 6 . Let $Y=Y_{1} \cup Y_{2}$ be a gadget and $F=K_{4}^{-}$be a component of $H^{\prime}$ disjoint from $Y$. If $e_{G}(Y, F) \geq 11$, then $G$ contains an $H$-approximation that is slightly better than $H^{\prime}$.

Before stating the last lemma, we need more notions. Let $H^{\prime}$ be a weak $H$-approximation. A half-gadget is a set $Z=\left\{z_{1}, z_{2}\right\} \subset V\left(H^{\prime}\right)$ formed either the two non-adjacent vertices of a $K_{4}^{-}$-component or by the two first (or last) vertices of a 6-lasso. For a half-gadget $Z=\left\{z_{1}, z_{2}\right\}$, a $Z$-attachment is a 5 -element subset $W$ of $V(G)$ whose vertices can be ordered $w_{1}, w_{2}, \ldots, w_{5}$ so that $w_{1} \in Z$, all the edges $w_{2} w_{3}, w_{3} w_{4}, w_{4} w_{5}, w_{5} w_{3}$ are in $E(G)$, and either $w_{2} \in Z$ or $w_{1} w_{2} \in E(G)$ (see Fig. 3).

We will use such attachments in Stages 3 and 4 to find subgraphs of $G$ that contain 7lassoes (when the half-gadget is a part of a $K_{4}^{-}$-component of $H^{\prime}$ ) and 9-lassoes (when the half-gadget is a part of a 6-lasso in $H^{\prime}$ ).

Lemma 5. Let $H^{\prime}$ be an $H$-approximation embedded into $G$. Let $T$ be the vertex set of a $K_{3}$-component of $H^{\prime}$, and $D$ be the vertex set of a component of $H^{\prime}$ with $H^{\prime}[D] \in$ 

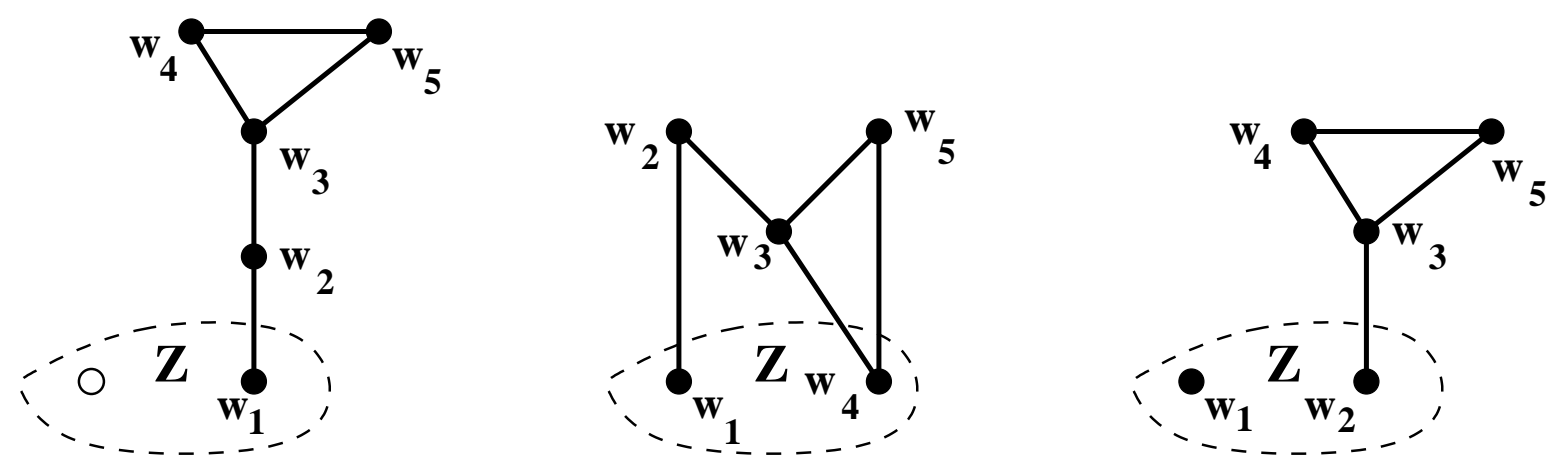

Figure 3. Three examples of $Z$-attachments. The white vertex on the left does not belong to the $Z$-attachment.

$\left\{K_{1}, K_{2}, K_{3}, K_{4}^{-}, C_{5}^{+}, D_{6}, D_{9}, D_{7}\right\}$ disjoint from $T$. Let $Z=\left\{z_{1}, z_{2}\right\} \subset V\left(H^{\prime}\right)$ be a halfgadget disjoint from $T \cup D$ and

$$
1.5 e_{G}(Z, D)+e_{G}(T, D)>4|D| .
$$

Then $Z \cup T \cup D$ contains a $Z$-attachment $W$ such that $G[Z \cup T \cup D-W]$ contains a copy of $H^{\prime}[D]$.

\section{Embedding SMall Lassoes}

Suppose that we have an embedding $\Psi$ into $G$ of an $H$-approximation $H_{1}$ whose components are in $\left\{K_{1}, K_{2}, K_{3}, K_{4}^{-}, C_{5}^{+}\right\}$. In this section we show how Stages 2, 3, and 4 work. After these stages, we will have an embedding into $G$ of an $H$-approximation whose components are larger: some of the components will be 6-lassoes, 9-lassoes, and 7-lassoes

Stage 2: Embedding of 6-lassoes. Each step of this stage starts from an $H$-approximation $H^{\prime}$ embedded into $G$ and finishes with an embedding into $G$ of a graph $H^{\prime \prime}$ that is obtained from $H^{\prime}$ by replacing two $K_{3}$-components from the same $M_{j}$ in $H^{\prime}$ with one 6 -lasso.

Suppose, by way of contradiction, that at some step, we have an embedding $\Psi$ into $G$ of an $H$-approximation $H^{\prime}$ whose components are $K_{1}$ 's, $K_{2}$ 's, $K_{3}$ 's, $K_{4}^{-}$'s, $C_{5}^{+}$'s, and 6-lassoes but cannot embed into $G$ any slightly better $H$-approximation. In other words, if $H^{\prime \prime}$ is obtained from $H^{\prime}$ by replacing two $K_{3}$-components with a 6-lasso, then $H^{\prime \prime}$ is not embeddable into $G$. Then $G$ has no edges between any two $K_{3}$-components of $H^{\prime}$.

Let some two $K_{3}$-components of $H^{\prime}$ in the same set $M_{j}$ have vertex sets $C_{1}=\left\{x_{1}, x_{2}, x_{3}\right\}$ and $C_{1}^{\prime}=\left\{x_{1}^{\prime}, x_{2}^{\prime}, x_{3}^{\prime}\right\}$. By $(1)$,

$e_{G}\left(C_{1} \cup C_{1}^{\prime}, V(G)-\left(C_{1} \cup C_{1}^{\prime}\right)\right)=\sum_{i=1}^{3}\left(d\left(x_{i}\right)+d\left(x_{i}^{\prime}\right)\right)-12 \geq 3 \sigma_{2}(G)-12 \geq 4 n-15>4(n-6)$.

So there is $D \subset V\left(H^{\prime}\right)$ such that $H^{\prime}[D]$ is a component of $H^{\prime}$ and

$$
e_{G}\left(C_{1} \cup C_{1}^{\prime}, D\right)>4|D| \text {. }
$$


If $H^{\prime}[D]=K_{3}$, then we are done. So, $H^{\prime}[D] \in\left\{K_{1}, K_{2}, K_{4}^{-}, C_{5}^{+}\right\}$. We will show that we can partition $C_{1} \cup C_{1}^{\prime} \cup D$ into two subsets $W_{1}$ and $W_{2}$ so that $G\left[W_{1}\right] \supseteq D_{6}$ and $G\left[W_{2}\right] \supseteq H^{\prime}[D]$. That would give an embedding into $G$ of a slightly better $H$-approximation.

This is easy when $|D|=1$. If $H^{\prime}[D]=K_{2}$, then $e_{G}\left(C_{1} \cup C_{1}^{\prime}, D\right) \geq 9$. We may assume that $e_{G}\left(C_{1}, D\right) \geq 5$. Then there exists $x \in C_{1}$ adjacent to both vertices in $D$. Let $X=C_{1}-x$ and $Z=C_{1}^{\prime} \cup D+x$. Since $e_{G}\left(C_{1}^{\prime}, D\right) \geq 9-6>0, G[Z]$ contains $D_{6}$.

Let $H^{\prime}[D]=K_{4}^{-}$. Then $e_{G}\left(C_{1} \cup C_{1}^{\prime}, D\right) \geq 17$. Suppose that a vertex $z \in D$ of degree 2 in $H^{\prime}[D]$ has at least two neighbors in $C_{1}$. In this case, if $D-z$ has a neighbor in $C_{1}^{\prime}$, then $G\left[C_{1}^{\prime} \cup D-z\right]$ contains a $D_{6}$ and $G\left[C_{1}+z\right]$ contains a $K_{4}^{-}$. Otherwise, $e_{G}\left(D-z, C_{1} \cup C_{1}^{\prime}\right) \leq 9$ and hence $e_{G}\left(D, C_{1} \cup C_{1}^{\prime}\right) \leq 15$, a contradiction to (5). So either of the 2-vertices in $H^{\prime}[D]$ has at most two neighbors in $C_{1} \cup C_{1}^{\prime}$ and thus $e_{G}\left(D, C_{1} \cup C_{1}^{\prime}\right) \leq 2 \cdot 2+2 \cdot 6=16$, a contradiction to (5) again.

Let $H^{\prime}[D]$ be a 6-lasso. If $C_{1}$ has a neighbor in one of the triangles of $H^{\prime}[D]$, then $C_{1}^{\prime}$ has no neighbors in the other triangle in $H^{\prime}[D]$. So $e_{G}\left(D, C_{1} \cup C_{1}^{\prime}\right) \leq 9+9<24=4|D|$, a contradiction to $(5)$.

Let $H^{\prime}[D]$ be a 5 -cycle $\left(y_{1}, y_{2}, y_{3}, y_{4}, y_{5}\right)$ with chord $y_{2} y_{5}$. First we prove that

$$
e_{G}\left(C_{1},\left\{y_{3}, y_{4}\right\}\right) \leq 3 \text { and } e_{G}\left(C_{1}^{\prime},\left\{y_{3}, y_{4}\right\}\right) \leq 3
$$

Indeed, if $e_{G}\left(C_{1},\left\{y_{3}, y_{4}\right\}\right) \geq 4$, then there is a matching of size two connecting $C_{1}$ and $\left\{y_{3}, y_{4}\right\}$. Thus $G\left[C_{1} \cup\left\{y_{3}, y_{4}\right\}\right]$ contains a $C_{5}^{+}$, and hence there is no edge between $C_{1}^{\prime}$ and $\left\{y_{1}, y_{2}, y_{5}\right\}$. Since $e_{G}\left(C_{1} \cup C_{1}^{\prime}, D\right) \geq 21$, all other edges between $D$ and $C_{1} \cup C_{1}^{\prime}$ are present. In particular, $G\left[C_{1}^{\prime}+y_{3}+y_{4}\right]$ contains a $C_{5}^{+}$and the subgraph of $G$ on the remaining 6 vertices contain a $D_{6}$. Thus, (6) holds.

We may assume that $e_{G}\left(C_{1}, D\right) \geq e_{G}\left(C_{1}^{\prime}, D\right)$. Then $e_{G}\left(C_{1}, D\right) \geq 11$. By $(6), e_{G}\left(C_{1},\left\{y_{1}, y_{2}, y_{5}\right\}\right) \geq$ 8. Let $x \in C_{1}$ be either the vertex non-adjacent to $y_{1}$ (if exists), or any vertex adjacent to both, $y_{2}$ and $y_{5}$. Then $G\left[C_{1}-x+y_{1}\right]$ is a $K_{3}$ and $G\left[D-y_{1}+x\right]$ contains a $C_{5}^{+}$. In order to avoid a $D_{6}$ in $G\left[C_{1}^{\prime} \cup C_{1}-x+y_{1}\right], e_{G}\left(y_{1}, C_{1}^{\prime}\right)=0$ and by $(6), e_{G}\left(C_{1}^{\prime}, D\right) \leq 6$. On the other hand, also by $(6), e_{G}\left(C_{1}, D\right) \leq 12$ and hence $e_{G}\left(C_{1}^{\prime}, D\right) \geq 9$, a contradiction to (5).

Stage 3: Embedding of 9-lassoes. We start from the $H$-approximation $H^{\prime}=H_{2}$ with given sets $M_{j}$ obtained at Stage 2. We will finish with an embedding into $G$ of an $H$-approximation $H_{3}$ such that each summand 3 in $\ell_{j} \geq 9$ corresponds to a part of a 9-lasso. Each step of the stage starts from an $H$-approximation $H^{\prime}$ embedded into $G$ and finishes with an embedding into $G$ of a graph $H^{\prime \prime}$ that is obtained from $H^{\prime}$ by replacing a $K_{3}$-component and a 6 -lasso from the same $M_{j}$ in $H^{\prime}$ with one 9-lasso. Suppose that at some step, we have an embedding $\Psi$ into $G$ of an $H$-approximation $H^{\prime}$ whose components are $K_{1}$ 's, $K_{2}$ 's, $K_{3}$ 's, $K_{4}^{-}$'s, $C_{5}^{+}$'s, and 6- and 9-lassoes but cannot embed into $G$ any slightly better $H$-approximation. In other words, if $H^{\prime \prime}$ is obtained from $H^{\prime}$ by replacing a $K_{3}$-component and a 6-lasso with a 9-lasso, then $H^{\prime \prime}$ is not embeddable into $G$. 
Let $T$ be the vertex set of a $K_{3}$-component in $H^{\prime}$ and $F$ be the vertex set of a 6 -lasso in $H^{\prime}$ containing path $\left(z_{1}, \ldots, z_{6}\right)$ and two chords $z_{1} z_{3}$ and $z_{4} z_{6}$.

Let $Z=\left\{z_{1}, z_{2}\right\}$. Then $E\left(\left\{z_{1}, z_{2}\right\}, T\right)=\emptyset$. So, by $(1), 1.5\left(d\left(z_{1}\right)+d\left(z_{2}\right)\right)+\sum_{u \in T} d(u) \geq$ $3 \sigma_{2}(G) \geq 4 n-3$. Vertices in $T \cup V(F)$ contribute at most $6+6$ to $\sum_{u \in T} d(u)$ and at most $2 \cdot 1.5 \cdot 5=15$ to $1.5\left(d\left(z_{1}\right)+d\left(z_{2}\right)\right)$. So, $1.5 e_{G}(Z, V(G-F)-T)+e_{G}(T, V(G-F)-T) \geq$ $4 n-3-12-15>4(n-9)$. Thus for some component of $H^{\prime}$ with vertex set, say $D$, $1.5 e_{G}(Z, D)+e_{G}(T, D)>4|D|$. Then $Z, T$ and $D$ satisfy the conditions of Lemma 5 . By this lemma, $Z \cup T \cup D$ contains a $Z$-attachment $W$ such that $G[Z \cup T \cup D-W]$ contains $H^{\prime}[D]$. Then $G[(F-Z) \cup W]$ contains a 9-lasso.

Stage 4: Embedding of 7-lassoes. We start from an embedding into $G$ of the $H$ approximation $H^{\prime}=H_{3}$ with given sets $M_{j}$ constructed at Stage 3 . We finish with an embedding into $G$ of an $H$-approximation $H_{4}$ such that to every $l_{j}=7$ corresponds a 7 -lasso. In each step, we find an embedding into $G$ of the graph $H^{\prime \prime}$ obtained from the starting graph $H^{\prime}$ by replacing a $K_{3}$-component and a $K_{4}^{-}$-component from the same $M_{j}$ with a 7-lasso.

Suppose that at some step, we have an embedding $\Psi$ into $G$ of an $H$-approximation $H^{\prime}$ whose components are $K_{1}$ 's, $K_{2}$ 's, $K_{3}$ 's, $K_{4}^{-}$'s, $C_{5}^{+}$'s, and 6-, 7-, and 9-lassoes but cannot embed into $G$ any slightly better $H$-approximation. In other words, if $H^{\prime \prime}$ is obtained from $H^{\prime}$ by replacing a $K_{3}$-component and a $K_{4}^{-}$-component with a 7-lasso, then $H^{\prime \prime}$ is not embeddable into $G$.

Let $T$ be the vertex set of a $K_{3}$-component in $H^{\prime}$ and $F$ be the vertex set of a $K_{4}^{-}$-component in $H^{\prime}$. Let $Z=\left\{z_{1}, z_{2}\right\}$ be the set of degree-2 vertices in $H^{\prime}[F]$. Then $E\left(\left\{z_{1}, z_{2}\right\}, T\right)=\emptyset$.

As in Stage 3, 1.5 $\left(d\left(z_{1}\right)+d\left(z_{2}\right)\right)+\sum_{u \in T} d(u) \geq 4 n-3$. Vertices in $T \cup V(D)$ contribute at most $6+6$ to $\sum_{u \in T} d(u)$ and at most $2 \cdot 1.5 \cdot 3=9$ to $1.5\left(d\left(z_{1}\right)+d\left(z_{2}\right)\right)$. So, $1.5 e_{G}(Z, V(G-$ $D)-T)+e_{G}(T, V(G-D)-T) \geq 4 n-3-12-9>4(n-7)$. Again, as in Stage 3, for some component of $H^{\prime}$ with vertex set, say $D, Z \cup T \cup D$ contains a $Z$-attachment $W$ such that $G[Z \cup T \cup D-W]$ contains $H^{\prime}[D]$. Then $G[(F-Z) \cup W]$ contains a 7-lasso.

\section{Final Embeddings and Proof of Theorem 5}

Recall that the components of the initial $H$-approximation $H^{\prime}$ at the beginning of Stage 5 are in the set $\left\{K_{1}, K_{2}, K_{3}, K_{4}^{-}, C_{5}, D_{6}, D_{7}, D_{9}\right\}$.

Stage 5: Embedding of $\ell_{j}$-lassoes for all $\ell_{j} \geq 6$. Let $k=l_{j}$. If $M_{j}$ (recall that $M_{j}$ is the set of building blocks of some component $R_{j}$ in $H$ ) does not consist of a $D_{k}$, then it contains some smaller components which are lassoes. We take pairs of components of $H^{\prime}$ in the same $M_{j}$ and try to embed into $G$ the graph $H^{\prime \prime}$ obtained from $H^{\prime}$ by replacing such a pair with one bigger double lasso. Suppose that at some step, we cannot proceed. Then by Stage 4, $k \geq 8$ and $k \neq 9$. Recall that the components in $M_{j}$ now are some double lassoes, and among them at most two $K_{4}^{-}$'s. Choose two such components with vertex sets $F_{1}$ and $F_{2}$. If $H^{\prime}\left[F_{i}\right]$ is not a $K_{4}^{-}$, then let $Y_{i}$ be the set of the two degree-2 vertices in one of the end triangles of $H^{\prime}\left[F_{i}\right]$. And if $H^{\prime}\left[F_{i}\right]$ is a $K_{4}^{-}$, then let $Y_{i}$ be the set of degree-2 vertices in $H^{\prime}\left[F_{i}\right]$. By the 
assumption, there are no edges between $Y_{1}$ and $Y_{2}$. Thus the degree sum of the four vertices in the gadget $Y_{1} \cup Y_{2}$ is at least $2 \sigma_{2}(G) \geq \frac{8 n-6}{3}$.

Suppose first that for an $i \in\{1,2\}$, some $y \in Y_{1}$ and $y^{\prime} \in Y_{2}$ have at least $\frac{4}{3}\left|F_{i}\right|-1$ neighbors in $F_{i}$. Then $H^{\prime}\left[F_{i}\right]$ is not a $K_{4}^{-}$, and thus is a double lasso $D_{t}$ for some $t \geq 6$. We may assume that $D_{t}$ consists of the path $\left(x_{1}=y, x_{2}, \ldots, x_{t}\right)$ with edges $x_{1} x_{3}$ and $x_{t-2} x_{t}$. To avoid a bigger double lasso, $y^{\prime}$ has no neighbors in $\left\{x_{1}, x_{2}, x_{t-1}, x_{t}\right\}$. Since $4 t / 3-1>t$, there exists $j$ with $4 \leq j \leq t-3$ such that $y x_{j}, y^{\prime} x_{j-1} \in E(G)$. So we have a bigger lasso $\left(H^{\prime}\left[F_{2}\right]-y^{\prime}, y^{\prime}, x_{j-1}, x_{j-2}, \cdots, y, x_{j}, \cdots, x_{t}\right)$, a contradiction.

If there are no such $i, y$ and $y^{\prime}$, then $e_{G}\left(Y, V(G)-F_{1}-F_{2}\right)>\frac{8}{3}\left|V(G)-F_{1}-F_{2}\right|$. So there is $D \subseteq V(G)-F_{1}-F_{2}$ such that $H^{\prime}[D]$ is a component of $H^{\prime}$ belonging to $\left\{K_{1}, K_{2}, K_{3}, K_{4}^{-}, C_{5}, D_{l}: l \geq 6\right\}$ and $e_{G}(Y, D)>\frac{8|D|}{3}$.

If $H^{\prime}[D]=K_{1}$ and $D=\{x\}$, then $e_{G}(x, Y) \geq 3$. So, $x$ has a neighbor $y$ in $Y_{1}$ and a neighbor $y^{\prime}$ in $Y_{2}$. For $y^{\prime \prime} \in Y_{2}-y^{\prime}$, graph $G\left[F_{1} \cup F_{2}+x-y^{\prime \prime}\right]$ contains a double lasso with $\left|F_{1} \cup F_{2}\right|$ vertices.

If $H^{\prime}[D]=K_{2}$ and $D=\left\{x_{1}, x_{2}\right\}$, then $e_{G}\left(\left\{x_{1}, x_{2}\right\}, Y\right) \geq 6$. By symmetry, we may assume that $e_{G}\left(\left\{x_{1}\right\}, Y\right) \geq 3$ and in particular that $Y_{1} \subset N_{G}\left(x_{1}\right)$. If some $y \in Y_{1}$ is adjacent to $x_{2}$, then $G\left[\left\{y, x_{2}\right\}\right]=K_{2}$ and $G\left[F_{1} \cup F_{2}+x_{1}-y\right]$ contains a double lasso with $\left|F_{1} \cup F_{2}\right|$ vertices. If $N\left(x_{2}\right) \cap Y_{1}=\emptyset$, then since $e_{G}\left(\left\{x_{1}, x_{2}\right\}, Y\right) \geq 6$, we have $Y \subset N_{G}\left(x_{1}\right)$ and $Y_{2} \subset N_{G}\left(x_{2}\right)$, so we have previous situation with $Y_{1}$ and $Y_{2}$ switched.

If $H^{\prime}[D]=K_{3}$ and $D=X=\left\{x_{1}, x_{2}, x_{3}\right\}$, then $e_{G}(X, Y) \geq 9$. By symmetry, we may assume that $e_{G}\left(X, Y_{1}\right) \geq 5, Y_{1}=\left\{y_{1}, y_{1}^{\prime}\right\}, N_{G}\left(y_{1}\right) \supset X$, and $\left|N_{G}\left(y_{1}^{\prime}\right) \cap X\right| \geq 2$. Since $e_{G}\left(X, Y_{2}\right) \geq 9-e_{G}\left(X, Y_{1}\right) \geq 3$ and $\left|N_{G}\left(y_{1}^{\prime}\right) \cap X\right| \geq 2$, some vertex $x \in N_{G}\left(y_{1}^{\prime}\right) \cap X$ has a neighbor in $Y_{2}$. So, $G\left[X-x+y_{1}\right]=K_{3}$ and $G\left[F_{1} \cup F_{2}+x-y_{1}^{\prime}\right]$ contains a double lasso with $\left|F_{1} \cup F_{2}\right|$ vertices.

If $H^{\prime}[D]$ is a $K_{4}^{-}$, then $e_{G}(Y, D)>32 / 3$, thus we apply Lemma 4 to get an $H$-approximation $H^{\prime \prime}$. If $H^{\prime}[D]$ is $D_{k}$ with $k \geq 6$, then $e_{G}(Y, D)>8 k / 3$, thus by Lemma 3 , there exists a $Y$ connector $Y^{\prime} \subset Y \cup D$ such that $G\left[(Y \cup D)-Y^{\prime}\right]$ contains a $D_{k}$. Thus we only need to consider the case when $H^{\prime}[D]$ is a $C_{5}$. By Lemma 2, there exists a $Y$-connector $Y^{\prime} \subset Y \cup D$ such that $G\left[(Y \cup D)-Y^{\prime}\right]$ either contains a $C_{5}$ or contains the double lasso $D_{5}$. If it contains a $C_{5}$, then we are done. So, suppose that $G$ contains the graph $H^{\prime \prime}$ obtained from $H^{\prime}$ by replacing $H^{\prime}\left[F_{1} \cup F_{2} \cup D\right]$ with a $\left(\left|F_{1}\right|+\left|F_{2}\right|\right)$-lasso and a 5-lasso $F_{3}$. Suppose that $F_{3}$ consists of the path $P=\left(x_{1}, x_{2}, x_{3}, x_{4}, x_{5}\right)$ plus edges $x_{1} x_{3}$ and $x_{3} x_{5}$. Let $Y_{1}=\left\{x_{1}, x_{2}\right\}, Y_{2}=\left\{x_{4}, x_{5}\right\}$ and $Y=Y_{1} \cup Y_{2}$. If at least one edge connecting $Y_{1}$ with $Y_{2}$ is present in $G$, then we are done. Otherwise, $H^{\prime \prime}$ is an $H$-quasi-approximation and $\sum_{v \in V\left(F_{3}\right)-x_{3}} d(v)>8 n / 3-2>$ $8(n-5) / 3+11$. Since the neighbors in $F_{3}$ of these vertices contribute only 8 to this sum, there is a component of $H^{\prime \prime}$ with vertex set, say, $F_{4}$ that contributes more than $8\left|F_{4}\right| / 3$ to this sum.

We now want to show that $Y \cup F_{4}$ contains a $Y$-connector $Y^{\prime}$ such that $G\left[Y \cup F_{4}-Y^{\prime}\right]$ contains $F_{4}$. That would imply that $G$ contains an $H$-approximation $H^{\prime \prime \prime}$ that is slightly better than $H^{\prime}$. Repeating the previous argument with the new $Y$ and with $F_{4}$ in place of 
$D$, we again reduce the problem to the case $F_{4}=C_{5}$. In this case, the last statement of Lemma 2 says that $G\left[F_{3} \cup F_{4}\right]$ contains two disjoint 5-cycles.

Stage 6: Embedding of $\ell_{j}$-cycles for all $\ell_{j} \geq 6$. At the beginning of the stage, every $M_{j}$ consists of one component, $D_{l_{j}}$. Suppose that at some step, we have an embedding into $G$ of an $H$-approximation $H^{\prime}$ but cannot embed any slightly better $H$-approximation. This means that for some $k \geq 6$, a component $H^{\prime}\left[F_{1}\right]$ of $H^{\prime}$ is the lasso $D_{k}$. Let $H^{\prime}\left[F_{1}\right]$ consist of the path $P=\left(y_{1}, y_{2}, \ldots, y_{k}\right)$ plus edges $y_{1} y_{3}$ and $y_{k-2} y_{k}$. Let $Y_{1}=\left\{y_{1}, y_{2}\right\}, Y_{2}=\left\{y_{k-1}, y_{k}\right\}$, and $Y=Y_{1} \cup Y_{2}$. If $G$ contains an edge connecting $Y_{1}$ with $Y_{2}$, then we are done. Otherwise, we repeat the argument for Stage 5 and $F_{1}=F_{2}$ with one additional possible situation for $F$ : it now can be also a cycle $C_{\ell}$ with $\ell \geq 6$. In this additional situation, Lemma 2 says that $G\left[F \cup C_{\ell}\right]$ contains a $C_{k}$ and a $C_{\ell}$, which gives up a slightly better $H$-approximation, a contradiction to the assumption.

\section{Proof of Lemma 3}

In this section, we will prove Lemma 3. Lemma 3 states that when there are a lot of edges between a gadget $Y$ and a lasso $D_{k}$, we are able to adjust them so that we will have a $Y$-connector and a new copy of $D_{k}$. Due to the edge density, $Y$ together with some part of $D_{k}$ form a dense subgraph, thus we are able to construct what we need.

Assume that $H^{\prime}[F]=D_{k}$ consists of a path $\left(u_{1}, u_{2}, \ldots, u_{k}\right)$ with the additional edges $u_{1} u_{3}$ and $u_{k-2} u_{k}$. Let $T_{1}=\left\{u_{1}, u_{2}, u_{3}\right\}, T_{2}=\left\{u_{k-2}, u_{k-1}, u_{k}\right\}$, and $P=\left\{u_{4}, \ldots, u_{k-3}\right\}$. Suppose that the lemma is false.

Claim 1. For $i=1,2, e_{G}\left(Y, T_{i}\right) \leq 8$. Furthermore, if $e_{G}\left(Y, T_{i}\right)=8$, then every vertex in $Y$ has a neighbor in $T_{i}$.

Proof. Suppose that $e_{G}\left(Y, T_{1}\right) \geq 9$. Then there exists $i \in\{1,2\}$ such that $e_{G}\left(Y_{i}, T_{1}\right) \geq 5$, and $e_{G}\left(Y_{3-i},\left\{u_{1}, u_{2}\right\}\right)>0$. By symmetry, we may assume that $u_{1} y_{3-i} \in E(G)$. Since $e_{G}\left(Y_{i}, T_{1}\right) \geq 5$, we can rename vertices $y_{i}$ and $y_{i}^{\prime}$ of $Y_{i}$ so that $u_{1} y_{i} \in E(G)$ and $\left(u_{2}, u_{3}, y_{i}^{\prime}\right)$ is a triangle. Then $\left(y_{i}, u_{1}, y_{3-i}\right)$ is a path in $G$ from $Y_{i}$ to $Y_{3-i}$, and $G\left[F-u_{1}+y_{i}^{\prime}\right]$ contains a $k$-lasso, a contradiction.

Now suppose that $e_{G}\left(Y, T_{1}\right)=8$ and that $y_{1} \in Y_{1}$ has no neighbors in $T_{1}$. Then the other three vertices of $Y$ have degree sequence 3,3,2 toward $T_{1}$ and one of $u_{1}$ and $u_{2}$, say $u_{1}$, is adjacent to all vertices in $Y-y_{1}$. Let $y_{2}$ be a vertex in $Y_{2}$ that has 3 neighbors in $T_{1}$. Then $Y-y_{2}+u_{1}$ is a $Y$-connector, and $G\left[F-u_{1}+y_{2}\right]$ contains a $D_{k}$.

Claim 2. Let $k \geq 9$. Let $S_{1}=\sum_{i=1}^{3} d\left(u_{i}, Y\right)+\frac{5}{6} d\left(u_{4}, Y\right)+\frac{1}{2} d\left(u_{5}, Y\right)+\frac{1}{6} d\left(u_{6}, Y\right)$ and $S_{2}=\frac{1}{6} d\left(u_{k-6}, Y\right)+\frac{1}{2} d\left(u_{k-5}, Y\right)+\frac{5}{6} d\left(u_{k-4}, Y\right)+\sum_{i=k-3}^{k} d\left(u_{i}, Y\right)$. Then $S_{1} \leq 12$ and $S_{2} \leq 12$.

Proof. Assume that $S_{1}>12$. By Claim 1, $\sum_{i=1}^{3} d\left(u_{i}, Y\right) \leq 8$.

CASE 1. $\quad \sum_{i=1}^{3} d\left(u_{i}, Y\right) \leq 7$. Then $\frac{5}{6} d\left(u_{4}, Y\right)+\frac{1}{2} d\left(u_{5}, Y\right)+\frac{1}{6} d\left(u_{6}, Y\right)>5$, that is, $5 d\left(u_{4}, Y\right)+3 d\left(u_{5}, Y\right)+d\left(u_{6}, Y\right) \geq 31$. 
If $d\left(u_{4}, Y\right)=4$, then $3 d\left(u_{5}, Y\right)+d\left(u_{6}, Y\right) \geq 11$. Since $d\left(u_{i}, Y\right) \leq 4$, (c3) yields that the degree sequence in $Y$ for $\left(u_{4}, u_{5}, u_{6}\right)$ is $(4,4,0)$. Then no vertex in $Y$ has a neighbor in $T_{1}$ (otherwise, we switch this vertex with $u_{4}$ ), and thus $\sum_{i=1}^{3} d\left(u_{i}, Y\right)=0$, and it implies that $S_{1}=32 / 5<12$, a contradiction.

If $d\left(u_{4}, Y\right) \leq 3$, then $3 d\left(u_{5}, Y\right)+d\left(u_{6}, Y\right) \geq 16$. So $d\left(u_{5}, Y\right)=d\left(u_{6}, Y\right)=4$ and $d\left(u_{4}, Y\right)=$ 3 , a contradiction to (c3).

CASE 2. $\quad \sum_{i=1}^{3} d\left(u_{i}, Y\right)=8$. Then $\frac{5}{6} d\left(u_{4}, Y\right)+\frac{1}{2} d\left(u_{5}, Y\right)+\frac{1}{6} d\left(u_{6}, Y\right)>4$, that is, $5 d\left(u_{4}, Y\right)+3 d\left(u_{5}, Y\right)+d\left(u_{6}, Y\right) \geq 25$.

If $d\left(u_{4}, Y\right) \leq 2$, then $3 d\left(u_{5}, Y\right)+d\left(u_{6}, Y\right) \geq 15$. Thus $d\left(u_{5}, Y\right)=4$ and $d\left(u_{6}, Y\right) \geq 3$, a contradiction to (c3).

If $d\left(u_{4}, Y\right)=3$, then $3 d\left(u_{5}, Y\right)+d\left(u_{6}, Y\right) \geq 10$. So, (c3) yields $\left(d\left(u_{5}, Y\right), d\left(u_{6}, Y\right)\right) \in$ $\{(2,4),(3,1),(3,2),(4,0),(4,1)\}$. In any case, since $d\left(u_{4}, Y\right)=3$ and $d\left(u_{5}, Y\right) \geq 2$, there is a neighbor $y \in Y$ of $u_{5}$ such that $Y-y+u_{4}$ is a $Y$-connector. By Claim 1, $y$ has a neighbor in $T_{1}$, and hence we may replace $u_{4}$ with $y$ in the double lasso, a contradiction.

If $d\left(u_{4}, Y\right)=4$, then $3 d\left(u_{5}, Y\right)+d\left(u_{6}, Y\right) \geq 5$. It follows that $d\left(u_{5}, Y\right) \geq 1$. Again, switch $y \in N\left(u_{5}\right) \cap Y$ with $u_{4}$, and we get a contradiction.

By Claim 1,

$$
e_{G}(Y, P)>\frac{8}{3}|P|=\frac{8}{3}(k-6)
$$

It $k=6$, then $P=\emptyset$, and hence $e_{G}(Y, P)=0$, a contradiction to $(7)$.

Let $k=7$, i.e., $|P|=1$. Then $e_{G}(Y, F) \geq 19$ and $e_{G}\left(Y, u_{4}\right) \geq 3$. By symmetry, we may assume that $y_{1}, y_{2}$, and $y_{1}^{\prime}$ are neighbors of $u_{4}$. Then $y_{1}$ and $y_{1}^{\prime}$ do not have neighbors in both $T_{1}$ and $T_{2}$. For the same reason, $y_{2}^{\prime}$ does not have neighbors in both $T_{1}$ and $T_{2}$. Hence there are at least $3 \times 3=9$ non-edges between $Y$ and $T_{1} \cup T_{2}$. Thus $y_{2}^{\prime}$ is a neighbor of $u_{4}$. It follows that $y_{2}$ does not have neighbors in both $T_{1}$ and $T_{2}$. We now have a contradiction to $e_{G}(F, Y) \geq 19$.

The next case is $k=8$, that is, $|P|=2$. Then $e_{G}(Y, F) \geq 22$ and $e_{G}\left(Y,\left\{u_{4}, u_{5}\right\}\right) \geq 6$. If $e_{G}\left(Y,\left\{u_{4}, u_{5}\right\}\right) \geq 7$, then $u_{4}$ and $u_{5}$ have at least three common neighbors in $Y$, and every common neighbor $y \in Y$ of $u_{4}$ and $u_{5}$ cannot have neighbors in $T_{1} \cup T_{2}$ (otherwise, we may switch $y$ with $u_{4}$ or $u_{5}$ to get a $Y$-connector and a $\left.D_{8}\right)$, thus $e_{G}(Y, F) \leq 32-3 \cdot 6=14$, a contradiction. If $e_{G}\left(Y,\left\{u_{4}, u_{5}\right\}\right)=6$, then the common neighbors of $u_{4}$ and $u_{5}$ in $Y$ have at least 9 non-edges to $T_{1} \cup T_{2}$, thus $e_{G}(Y, F) \leq 32-9-2=21$, a contradiction.

If $k=9$, then by Claim $2, e_{G}(Y, F) \leq 24=8 / 3|F|$, a contradiction.

Now we let $k \geq 10$. Consider the sum

$$
S=S_{1}+\frac{1}{6} \sum_{i=5}^{k-5}\left(d_{3}\left(u_{i}, Y\right)+d_{3}\left(u_{i+1}, Y\right)\right)+S_{2} .
$$


Observe that $S=e_{G}(F, Y)>\frac{8}{3} k$. This and Claim 2 imply that $\sum_{i=5}^{k-5}\left(d_{3}\left(u_{i}, Y\right)+\right.$ $\left.d_{3}\left(u_{i+1}, Y\right)\right)>6(8 / 3 k-24)=16(k-9)$. Then there exists $i$ with $5 \leq i \leq k-5$ such that $d_{3}\left(u_{i}, Y\right)+d_{3}\left(u_{i+1}, Y\right) \geq 17$. By the symmetry between $u_{i}$ and $u_{i+1}$, we may assume that $d_{3}\left(u_{i}, Y\right) \geq 9$. Now statement (c4) of Lemma 1 yields that one of the six configurations in Figure 1 occurs.

Consider $P$ as the union of three paths with the vertex sets $P_{1}=\left\{u_{4}, \ldots, u_{i-1}\right\}, P_{0}=$ $\left\{u_{i}, u_{i+1}\right\}$, and $P_{2}=\left\{u_{i+2}, \ldots, u_{k-3}\right\}$.

Claim 3. Let $k \geq 10$. Suppose that vertices $y_{1}, y_{1}^{\prime} \in Y_{1}$ and $y_{2}, y_{2}^{\prime} \in Y_{2}$ are chosen so that (R1) $y_{1}^{\prime} u_{i}, y_{2}^{\prime} u_{i+1} \in E(G)$ and

(R2) $y_{1} u_{i-1}, y_{2} u_{i+2} \in E(G)$.

Then

(S1) for each $5 \leq j \leq i-1$, if $u_{j} y_{2} \in E(G)$, then $u_{j-1} y_{1} \notin E(G)$, and if $u_{4} y_{2} \in E(G)$, then $y_{1}$ has no neighbors in $T_{1}$;

(S2) similarly, for each $i+2 \leq j \leq k-4$, if $u_{j} y_{1} \in E(G)$, then $u_{j+1} y_{2} \notin E(G)$, and if $u_{k-3} y_{1} \in E(G)$, then $y_{2}$ has no neighbors in $T_{2}$;

(S3) $d\left(y_{1}, P_{1}\right)+d\left(y_{2}, P_{1}\right)+e_{G}\left(Y, T_{1}\right) \leq i+4$ and $d\left(y_{1}, P_{2}\right)+d\left(y_{2}, P_{2}\right)+e_{G}\left(Y, T_{2}\right) \leq k-i+4$.

Proof. Suppose first that $u_{j} y_{2} \in E(G)$ and $u_{j-1} y_{1} \in E(G)$ for some $5 \leq j \leq i-1$. Then by (R2), the sequence

$$
\left(T_{2}, u_{k-4}, u_{k-5}, \ldots, u_{i+2}, y_{2}, u_{j}, u_{j+1}, \ldots, u_{i-1}, y_{1}, u_{j-1}, u_{j-2}, \ldots, u_{4}, T_{1}\right)
$$

forms a double lasso of order $k$ in $G$, and by (R1), $Y-y_{1}-y_{2}+u_{i}+u_{i+1}$ is a $Y$-connector. The same argument proves the second part of (S1), and a symmetric argument proves (S2).

By $(\mathrm{S} 1), d\left(y_{1}, P_{1}\right)+d\left(y_{2}, P_{1}\right) \leq i-3$. Moreover, the equality is attained only if $i-4$ is odd and $y_{1}$ and $y_{2}$ are both adjacent to $u_{4}, u_{6}, \ldots, u_{i-1}$. Then again by $(S 1), y_{1}$ is not adjacent to $T_{1}$. Therefore, by Claim $1, e_{G}\left(Y, T_{1}\right) \leq 7$. This proves the first part of (S3). The proof of the other part is analogous.

Consider configurations (A), (C) and (E) in Figure 1. For each choice of $y_{1} \in Y_{1}$ and $y_{2} \in Y_{2}$ in these configurations, both (R1) and (R2) hold. So, by Claim 3, (S1) and (S2) hold for each such choice. In particular, if $u_{4}$ (respectively, $u_{k-3}$ ) has a neighbor in $Y_{2}$ (respectively, $Y_{1}$ ), then there are no edges between $T_{1}$ and $Y_{1}$ (respectively, $T_{2}$ and $Y_{2}$ ) which yields $e_{G}\left(Y, T_{1}\right) \leq 6$ (respectively, $\left.e_{G}\left(Y, T_{2}\right) \leq 6\right)$. It follows from (S1) and (S2) that

$$
\begin{gathered}
e_{G}(Y, F)=e_{G}\left(Y,\left\{u_{1}, \ldots, u_{i-1}\right\}\right)+e_{G}\left(Y,\left\{u_{i}, u_{i+1}\right\}\right)+e_{G}\left(Y,\left\{u_{i+2}, \ldots, u_{k}\right\}\right) \leq \\
(2+2(i-1))+6+(2+2(k-i-1))=2 k+6 .
\end{gathered}
$$

Since $e_{G}(Y, F)>8 k / 3$, we get $2 k+6>8 k / 3$, i.e., $k<9$, a contradiction.

Consider now configuration (D) in Fig. 1. The set $Y-y_{1}-y_{2}^{\prime}+u_{i}+u_{i+1}$ is a $Y$-connector. Graph $G\left[F-u_{i}-u_{i+1}+y_{1}\right]$ contains lasso $D_{k-1}$. So, if $y_{2}^{\prime}$ is adjacent to two consecutive vertices in $P_{1}$ or $P_{2}$, then $G\left[F-u_{i}-u_{i+1}+y_{1}+y_{2}^{\prime}\right]$ contains lasso $D_{k}$, a contradiction. Thus 
$d\left(y_{2}^{\prime}, P_{1}\right) \leq(i-4+1) / 2$ and $d\left(y_{2}^{\prime}, P_{2}\right) \leq(k-i-4+1) / 2$. Since $y_{2}^{\prime} u_{i} \notin E(G)$, we obtain $d\left(y_{2}^{\prime}, P\right) \leq k / 2-2$. Since $y_{1}^{\prime} u_{i+2} \notin E(G)$, we have $d\left(y_{1}^{\prime}, P\right) \leq k-7$. Together with (S3) and the fact that $e_{G}\left(\left\{y_{1}, y_{2}\right\}, P_{0}\right)=2$ we obtain

$$
e_{G}(Y, F) \leq(k / 2-2)+(k-7)+(i+4)+(k-i+4)+2=5 k / 2+1 .
$$

It follows that $5 k / 2+1>8 k / 3$, i.e., $k<6$, a contradiction.

Consider configuration (F) in Fig. 1. The situation here is symmetric to (D). The set $Y-y_{1}^{\prime}-y_{2}+u_{i}+u_{i+1}$ is a $Y$-connector. Graph $G\left[F-u_{i}-u_{i+1}+y_{2}\right]$ contains lasso $D_{k-1}$. Vertex $y_{1}^{\prime}$ has no two consecutive neighbors on $P_{1}$ and $P_{2}$. Since $y_{1}^{\prime} u_{i+2} \notin E(G)$, $d\left(y_{1}^{\prime}, P_{2}\right) \leq(k-i-4) / 2, d\left(y_{1}^{\prime}, P_{1}\right) \leq(i-3) / 2$, and hence $d\left(y_{1}^{\prime}, P\right) \leq(k-3) / 2$. Since $y_{2}^{\prime} u_{i}, y_{2}^{\prime} u_{i+2} \notin E(G)$, we have $d\left(y_{2}^{\prime}, P\right) \leq k-8$. Together with (S3) and the fact that $e_{G}\left(\left\{y_{1}, y_{2}\right\}, P_{0}\right)=3$ we obtain

$$
e_{G}(Y, F) \leq(k-3) / 2+(k-8)+(i+4)+(k-i+4)+3=(5 k+3) / 2,
$$

which yields $k<9$, a contradiction.

Finally, consider configuration (B) in Fig. 1. Again, the set $Y-y_{1}^{\prime}-y_{2}+u_{i}+u_{i+1}$ is a $Y$-connector and $G\left[F-u_{i}-u_{i+1}+y_{2}\right]$ contains lasso $D_{k-1}$. Since $y_{1}^{\prime} u_{i+2}, y_{1}^{\prime} u_{i-1} \notin E(G)$ and $y_{1}^{\prime}$ has no two consecutive neighbors on $P_{1}$ and $P_{2}$, we have $d\left(y_{1}^{\prime}, P_{1}\right) \leq\lfloor(i-4) / 2\rfloor$ and $d\left(y_{1}^{\prime}, P_{2}\right) \leq\lfloor(k-i-4) / 2\rfloor$. Since $y_{2}^{\prime} u_{i} \notin E(G)$, we have $d\left(y_{2}^{\prime}, P\right) \leq k-7$. Together with (S3) and the fact that $e_{G}\left(\left\{y_{1}, y_{2}\right\}, P_{0}\right)=3$ we obtain

$$
e_{G}(Y, F) \leq(\lfloor(i-4) / 2\rfloor+\lfloor(k-i-4) / 2\rfloor+2)+(k-7)+(i+4)+(k-i+4)+3 \leq 5 k / 2+2,
$$

which yields $k<12$. Furthermore, if $k \in\{10,11\}$, then either $i=5$ (and hence $\lfloor(i-4) / 2\rfloor=$ $(i-5) / 2)$ or $k-i=5$ (and hence $\lfloor(k-i-4) / 2\rfloor=(k-i-5) / 2)$. In both cases, by (8), $e_{G}(Y, F) \leq(5 k+3) / 2$, which yields $k<9$.

\section{Proof of Lemma 4}

In this section, we prove Lemma 4 . Lemma 4 roughly states that if there are a lot of edges between a gadget $Y$ and some component $F=K_{4}^{-}$, then we are able to adjust them to get a $Y$-connector and a new copy of $K_{4}^{-}$. We will see that sometimes it is not enough to adjust only the union of the gadget and $K_{4}^{-}$, and we need to consider another component $D$ which has a lot of edges to $Y \cup F$.

Let $F=\left\{w_{1}, w_{2}, w_{3}, w_{4}\right\}$ and $H^{\prime}[F]=K_{4}^{-}$be such that $d_{H^{\prime}}\left(w_{1}\right)=d_{H^{\prime}}\left(w_{2}\right)=2$ and $e_{G}(Y, F) \geq 11$. In terms of the complement, this means that

$$
e_{\bar{G}}(Y, F) \leq 5 .
$$

Suppose by contradiction that the lemma is not true for $Y$ and $F$.

Assume first that for some $i \in\{1,2\}, w_{i}$ has neighbors in both, $Y_{1}$ and $Y_{2}$. By symmetry, we may assume that $w_{1}$ is adjacent to $y_{1}$ and $y_{2}$. If some $y \in\left\{y_{1}^{\prime}, y_{2}^{\prime}\right\}$ has at least two neighbors in $\left\{w_{2}, w_{3}, w_{4}\right\}$, then $G\left[\left\{y, w_{2}, w_{3}, w_{4}\right\}\right]$ contains $K_{4}^{-}$, and $Y-y+w_{1}$ is a $Y$-connector. So, $e_{\bar{G}}\left(\left\{y_{1}^{\prime}, y_{2}^{\prime}\right\},\left\{w_{2}, w_{3}, w_{4}\right\}\right) \geq 4$. By $(9), w_{1}$ has a neighbor in $\left\{y_{1}^{\prime}, y_{2}^{\prime}\right\}$. By symmetry, we may 
assume that $y_{1}^{\prime} w_{1} \in E(G)$. Then, as above, $y_{1}$ has at most one neighbor in $\left\{w_{2}, w_{3}, w_{4}\right\}$. Hence $e_{\bar{G}}(Y, F) \geq 6$, a contradiction. Thus we may assume that $w_{1}$ has no neighbors in $Y_{2}$, and $w_{2}$ has no neighbors in some $Y_{j}$, where $j \in\{1,2\}$.

If $j=2$, then again by (9), $w_{1}$ and $w_{2}$ have a common neighbor, say $y_{1}$, in $Y_{1}$. Also by (9), at most one edge between $Y_{1}$ and $\left\{w_{3}, w_{4}\right\}$ is missing. So, by symmetry, we can assume that $y_{1} w_{3}, y_{1}^{\prime} w_{4} \in E(G)$. Then $G\left[V(F)-w_{4}+y_{1}\right]$ contains $K_{4}^{-}$and $Y-y_{1}+w_{4}$ is a $Y$-connector. This contradiction proves that $j=1$. Furthermore, if $w_{1} w_{2} \in E(G)$, then we can switch the roles of $\left\{w_{1}, w_{2}\right\}$ and $\left\{w_{3}, w_{4}\right\}$ thus forcing $e_{\bar{G}}(Y, H) \geq 8$, a contradiction.

So, from now on, $E^{\prime}=\left\{w_{1} w_{2}, w_{1} y_{2}, w_{1} y_{2}^{\prime}, w_{2} y_{1}, w_{2} y_{1}^{\prime}\right\} \subseteq E(\bar{G})$. Let $F_{1}=F \cup Y$. By (9) and symmetry, we may assume that the only non-edge of $G\left[F_{1}\right]$ that is not in $E^{\prime}$ (if exists) is either $y_{1} w_{1}$ or $y_{1} w_{3}$ (see Figure 4 ). So, if the $Y_{i}$-block is a $K_{4}^{-}$, then we can switch the roles of $F$ and this block. This implies that

$$
\text { neither } Y_{1} \text {-block nor } Y_{2} \text {-block is a } K_{4}^{-} \text {. }
$$

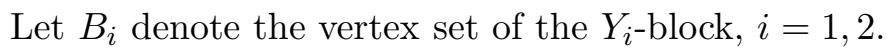
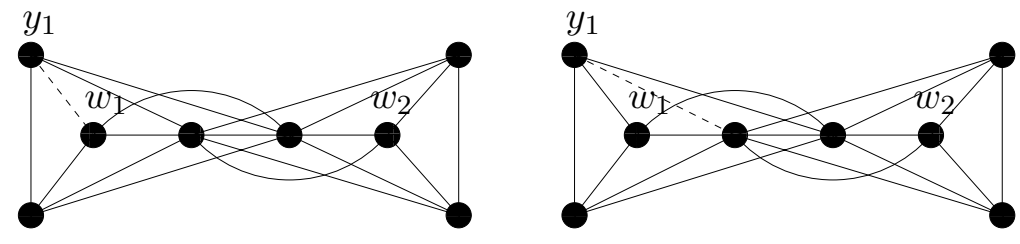

FiguRE 4. The subgraph induced by $F_{1}=F \cup Y$.

Let $S=Y \cup\left\{w_{1}, w_{2}\right\}$. By (2),

$$
\sum_{v \in S} d(v) \geq 3 \sigma_{2}(G) \geq 4 n-3
$$

Since the sum gains at most 24 from the neighbors in $F_{1}$,

$$
e_{G}\left(S, V(G)-F_{1}\right) \geq 4 n-27=4(n-8)+5 .
$$

Therefore, either there exists a component $H^{\prime}[D]$ of $H^{\prime}$ such that

$$
e_{G}(S, D)>4|D|,
$$

or for some $i \in\{1,2\}$ the set $D=B_{i}-Y_{i}$ satisfies (12), or $B_{1}=B_{2}$ and the set $D=B_{1}-Y$ satisfies (12).

If $H^{\prime}[D]=K_{1}$ is a component of $H^{\prime}$ and $D=\{u\}$, then by (12) there are at least 5 edges from $u$ to $S$. So, $u$ has a neighbor in $Y_{1}$ and a neighbor, say $y_{2}$ in $Y_{2}$. Then $Y-y_{2}^{\prime}+u$ is a $Y$-connector and $\left\{y_{2}^{\prime}\right\}$ forms a new $K_{1}$-component of $H^{\prime}$ (see Figure 5).

If $H^{\prime}[D]$ is a $K_{2}$-component of $H^{\prime}$ and $D=\left\{u_{1}, u_{2}\right\}$, then by $(12), e_{G}(D, S) \geq 9$ and so $e_{G}(D, Y) \geq 5$. By symmetry, we may assume that $e_{G}\left(u_{1}, Y\right) \geq 3$. We will construct a $Y$-connector without using $F$, so we may assume that $N\left(u_{1}\right) \supset Y_{1}+y_{2}$. If $u_{2}$ is adjacent to some $y \in Y_{1}+y_{2}$, then $G\left[\left\{y, u_{2}\right\}\right]=K_{2}$ and $Y-y+u_{1}$ is a $Y$-connector. Otherwise, since 


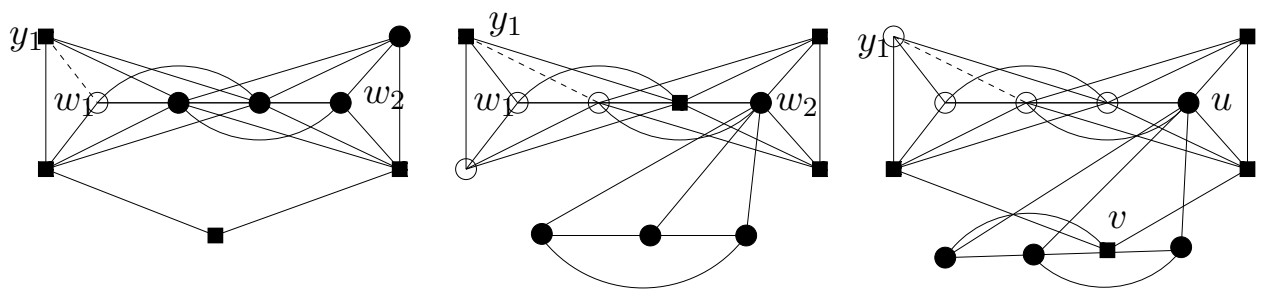

Figure 5. Partitions with $K_{1}, K_{3}$ and $K_{4}^{-}$

$e_{G}(D, Y) \geq 5$, we have $N\left(u_{1}\right) \supset Y$ and $u_{2}$ has a neighbor $y \in Y$. So, again $G\left[\left\{y, u_{2}\right\}\right]=K_{2}$ and $Y-y+u_{1}$ is a $Y$-connector.

Suppose that $H^{\prime}[D]=K_{3}$ is a component of $H^{\prime}$ and $D=\left\{u_{1}, u_{2}, u_{3}\right\}$. By $(12), e_{G}(S, D) \geq$ 13. If for some $i \in\{1,2\},\left|D \cap N\left(w_{i}\right)\right| \geq 2$, then $G\left[D+w_{i}\right]$ contains a $K_{4}^{-}, G\left[\left\{y_{3-i}, w_{3-i}, w_{3}\right\}\right]=$ $K_{3}$, and $Y-y_{3-i}+w_{4}$ is a $Y$-connector. Otherwise, $e_{G}(Y, D) \geq 13-2=11$. It follows that $G\left[D+y_{1}\right]$ contains a $K_{4}^{-},\left\{y_{1}^{\prime}, w_{1}, w_{3}, y_{2}^{\prime}\right\}$ is a $Y$-connector, and $G\left[\left\{w_{2}, w_{4}, y_{2}\right\}\right]=K_{3}$.

Suppose that $H^{\prime}[D]=K_{4}^{-}$is a component of $H^{\prime}$ and $D=\left\{u_{1}, u_{2}, u_{3}, u_{4}\right\}$, where $u_{1} u_{2} \notin$ $E\left(H^{\prime}\right)$. By $(12), e_{G}(Y, D)>4|D|-e_{G}\left(\left\{w_{1}, w_{2}\right\}, D\right) \geq 2|D|$. Then some $u \in D$ has at least 3 neighbors in $Y$ and hence has a neighbor $y \in Y_{1}$ and a neighbor $y^{\prime} \in Y_{2}$. Let $y^{\prime \prime} \in Y_{2}-y^{\prime}$. If $e_{G}(Y, D) \leq 10$, then by $(12), e_{G}\left(\left\{w_{1}, w_{2}\right\}, D\right) \geq 7$, and by symmetry we may assume that $e_{G}\left(w_{1}, D\right)=4$. Then $Y-y^{\prime \prime}+u$ is a $Y$-connector, and each of $G\left[F-w_{1}+y^{\prime \prime}\right]$ and $G\left[D-u+w_{1}\right]$ contains $K_{4}^{-}$. So, suppose that $e_{G}(Y, D) \geq 11$. Then repeating our argument for $F$, we may assume that the missing edges in $E(Y, D)$ are $u_{1} y_{2}, u_{1} y_{2}^{\prime}, u_{2} y_{1}, u_{2} y_{1}^{\prime}$, and maybe one more edge. In particular, $e_{G}(Y, D) \leq 12$ and hence $e_{G}\left(\left\{w_{1}, w_{2}\right\}, D\right) \geq 5$. If $w_{2} u_{1} \in E(G)$, let $y$ be a neighbor of $u_{1}$ in $Y_{1}$ and $y^{\prime} \in Y_{1}-y$. In this case, $\left\{y_{2}, w_{2}, u_{1}, y\right\}$ is a $Y$-connector, and each of $G\left[F-w_{2}+y_{2}^{\prime}\right]$ and $G\left[D-u_{1}+y^{\prime}\right]$ contains a $K_{4}^{-}$. So, $w_{2} u_{1} \notin E(G)$ and similarly $w_{1} u_{2} \notin E(G)$. Thus, since $e_{G}\left(\left\{w_{1}, w_{2}\right\}, D\right) \geq 5$, either $e_{G}\left(w_{1}, D-u_{2}\right)=3$ or $e_{G}\left(w_{2}, D-u_{1}\right)=3$. If $e_{G}\left(w_{1}, D-u_{2}\right)=3$, then let $h \in\{3,4\}$ be such that $u_{h} y_{1} \in E(G)$ and let $y \in Y_{2}$ be adjacent to $u_{2}$ and $y^{\prime} \in Y_{2}-y$. In this notation, $\left\{y_{1}, u_{h}, u_{2}, y\right\}$ is a $Y$-connector, and each of $G\left[F-w_{1}+y^{\prime}\right]$ and $G\left[\left\{w_{1}, y_{1}^{\prime}, u_{1}, u_{7-h}\right\}\right]$ contains a $K_{4}^{-}$. If $e_{G}\left(w_{1}, D-u_{2}\right)<3$, then $e_{G}\left(w_{2}, D-u_{1}\right)=3$ and $e_{G}(Y, D)=12$, so that the missing edges in $E(Y, D)$ are only $u_{1} y_{2}, u_{1} y_{2}^{\prime}, u_{2} y_{1}$, and $u_{2} y_{1}^{\prime}$. Then $\left\{y_{1}, u_{1}, u_{3}, y_{2}\right\}$ is a $Y$-connector, and each of $G\left[F-w_{2}+y_{1}^{\prime}\right]$ and $G\left[\left\{w_{2}, y_{2}^{\prime}, u_{2}, u_{4}\right\}\right]$ contains a $K_{4}^{-}$.

Before considering the remaining cases, we need two facts.

Lemma 6. Let $P=\left(u_{1}, u_{2}, u_{3}, u_{4}\right)$ be a path in $G-F_{1}$ and $U=\left\{u_{1}, u_{2}, u_{3}, u_{4}\right\}$. If

$$
d_{3}\left(u_{2}, S\right)+d_{3}\left(u_{3}, S\right) \geq 25,
$$

then $F_{1} \cup U$ can be partitioned into three sets, $W_{1}, W_{2}$, and $W_{3}$ so that $W_{1}$ is a $Y$-connector, $G\left[W_{2}\right]$ contains a $u_{1}, u_{4}$-path of length 3 , and $G\left[W_{3}\right]$ contains a $K_{4}^{-}$.

Proof. Case 1: $d_{3}\left(u_{2},\left\{w_{1}, w_{2}\right\}\right)+d_{3}\left(u_{3},\left\{w_{1}, w_{2}\right\}\right) \geq 9$. We need two claims. 
Claim 4. Let $j \in\{2,3\}$.

(a) If $w_{1} u_{j-1}, w_{1} u_{j+1}, w_{2} u_{j} \in E(G)$, then $y_{1}, y_{2}$, and $y_{2}^{\prime}$ are not neighbors of $u_{j}$.

(b) If $w_{2} u_{j-1}, w_{2} u_{j+1}, w_{1} u_{j} \in E(G)$, then $y_{1}^{\prime}, y_{2}$, and $y_{2}^{\prime}$ are not neighbors of $u_{j}$.

Proof. Let $j=3$ (the case $j=2$ is symmetric). Suppose that $w_{1} u_{2}, w_{1} u_{4}, w_{2} u_{3} \in E(G)$. If $z \in Y_{2}$ is a neighbor of $u_{3}$, then $Y-z+w_{4}$ is a $Y$-connector, $G\left[\left\{w_{3}, w_{2}, u_{3}, z\right\}\right]$ contains a $K_{4}^{-}$, and $\left(u_{1}, u_{2}, w_{1}, u_{4}\right)$ is a path in $G$. If $y_{1} u_{3} \in E(G)$, then $Y-y_{1}^{\prime}-y_{2}+u_{3}+w_{2}$ is a $Y$-connector, $G\left[\left\{y_{1}^{\prime}, w_{3}, w_{4}, y_{2}^{\prime}\right\}\right]=K_{4}^{-}$, and $\left(u_{1}, u_{2}, w_{1}, u_{4}\right)$ is a path in $G$. This proves (a).

Suppose now that $w_{2} u_{2}, w_{2} u_{4}, w_{1} u_{3} \in E(G)$. Let $w=y_{1}$ and $w^{\prime}=y_{1}^{\prime}$ if $y_{1} w_{1} \in E(G)$ and $w=y_{1}^{\prime}$ and $w^{\prime}=y_{1}$ otherwise. If $z \in Y_{2}$ is a neighbor of $u_{3}$ and $z^{\prime} \in Y_{2}-z$, then $Y-w^{\prime}-z^{\prime}+u_{3}+w_{1}$ is a $Y$-connector, $G\left[\left\{w^{\prime}, w_{3}, w_{4}, z^{\prime}\right\}\right]=K_{4}^{-}$, and $\left(u_{1}, u_{2}, w_{2}, u_{4}\right)$ is a path in $G$. If $y_{1}^{\prime} u_{3} \in E(G)$, then $Y-y_{1}^{\prime}+w_{4}$ is a $Y$-connector, $G\left[\left\{w_{1}, w_{3}, u_{3}, y_{1}^{\prime}\right\}\right]$ contains a $K_{4}^{-}$, and $\left(u_{1}, u_{2}, w_{2}, u_{4}\right)$ is a path in $G$.

Claim 5. Let $h \in\{1,4\}$.

(a) If $w_{1} u_{2}, w_{1} u_{3}, w_{2} u_{h} \in E(G)$, then either $e_{G}\left(u_{5-h}, Y_{2}\right)=0$ or $e_{G}\left(y_{1}^{\prime},\left\{u_{2}, u_{3}\right\}\right)=0$.

(b) If $w_{2} u_{2}, w_{2} u_{3}, w_{1} u_{h} \in E(G)$, then either $u_{5-h} y_{1}^{\prime} \notin E(G)$ or $e_{G}\left(Y_{2},\left\{u_{2}, u_{3}\right\}\right)=0$.

Proof. By symmetry, we consider only $h=1$. Assume first that $w_{1} u_{2}, w_{1} u_{3}, w_{2} u_{1} \in E(G)$, $e_{G}\left(u_{4}, Y_{2}\right)>0$, and $e_{G}\left(y_{1}^{\prime},\left\{u_{2}, u_{3}\right\}\right)>0$. Let $z$ be a neighbor of $u_{4}$ in $Y_{2}$. Then $Y-y_{1}^{\prime}-z+$ $w_{3}+w_{4}$ is a $Y$-connector, $G\left[\left\{y_{1}^{\prime}, w_{1}, u_{2}, u_{3}\right\}\right]$ contains a $K_{4}^{-}$, and $\left(u_{1}, w_{2}, z, u_{4}\right)$ is a path in $G$. This proves (a).

Similarly, assume that $w_{2} u_{2}, w_{2} u_{3}, w_{1} u_{1} \in E(G), u_{4} y_{1}^{\prime} \in E(G)$, and $e_{G}\left(Y_{2},\left\{u_{2}, u_{3}\right\}\right)>0$. Let $z$ be a vertex in $Y_{2}$ adjacent to either $u_{2}$ or $u_{3}$. Then $Y-y_{1}^{\prime}-z+w_{3}+w_{4}$ is a $Y$-connector, $G\left[\left\{\left\{z, w_{2}, u_{2}, u_{3}\right\}\right]\right.$ contains a $K_{4}^{-}$, and $\left(u_{1}, w_{1}, y_{1}^{\prime}, u_{4}\right)$ is a path in $G$.

Case 1.1: $e_{G}\left(\left\{u_{2}, u_{3}\right\},\left\{w_{1}, w_{2}\right\}\right)=3$. To have $d_{3}\left(u_{2},\left\{w_{1}, w_{2}\right\}\right)+d_{3}\left(u_{3},\left\{w_{1}, w_{2}\right\}\right) \geq 9$, we need $e_{G}\left(\left\{u_{1}, u_{4}\right\},\left\{w_{1}, w_{2}\right\}\right) \geq 3$. Thus there is a matching of size 2 between $\left\{w_{1}, w_{2}\right\}$ and $\left\{u_{1}, u_{4}\right\}$, so by symmetry, we may assume that $w_{1} u_{1}, w_{2} u_{4} \in E(G)$.

If $w_{1} u_{3}, w_{2} u_{2} \in E(G)$, then by Claim $4, y_{1}, y_{2}, y_{2}^{\prime}$ are not neighbors of $u_{2}$ and $u_{3}$, we have a contradiction to $d_{3}\left(u_{2}, S\right)+d_{3}\left(u_{3}, S\right) \geq 25$. So we assume that either $w_{1} u_{3} \notin E(G)$ or $w_{2} u_{2} \notin E(G)$ (thus $w_{1} u_{2}, w_{2} u_{3} \in E(G)$ ). If $u_{1} w_{2} \in E(G)$ and $u_{4} w_{1} \in E(G)$, then again by Claim $4, y_{1}, y_{2}, y_{2}^{\prime}$ are not neighbors of $u_{2}$ and $u_{3}$, we have a contradiction to (13). Thus, exactly one of $w_{1} u_{3}, w_{2} u_{2}$ is an edge in $G$ and exactly one of $u_{1} w_{2}, u_{4} w_{1}$ is an edge in $G$. So, we have four possibilities.

If $w_{2} u_{1}, w_{2} u_{2} \in E(G)$, then by Claim 4 (a), $y_{1}^{\prime}, y_{2}, y_{2}^{\prime}$ are not neighbors of $u_{2}$. So by (13), $e_{G}\left(u_{3}, Y_{1}\right), e_{G}\left(u_{3}, Y_{2}\right) \geq 1$. Let $u_{3} y_{2} \in E(G)$. Then $Y-y_{2}^{\prime}+u_{3}$ is a $Y$-connector, $\left(u_{1}, u_{2}, w_{2}, u_{4}\right)$ is a path in $G$, and $G\left[w_{1}, w_{3}, w_{4}, y_{2}^{\prime}\right]$ contains a $K_{4}^{-}$.

Symmetrically, if $w_{1} u_{3}, w_{1} u_{4} \in E(G)$, then by Claim 4 (b), $y_{1}, y_{2}, y_{2}^{\prime}$ are not neighbors of $u_{3}$. So by $(13), e_{G}\left(u_{2}, Y_{1}\right), e_{G}\left(u_{2}, Y_{2}\right) \geq 1$, and we may assume that $u_{2} y_{2} \in E(G)$. Then 
$Y-y_{2}^{\prime}+u_{2}$ is a $Y$-connector, $\left(u_{1}, w_{1}, u_{3}, u_{4}\right)$ is a path in $G$, and $G\left[w_{2}, w_{3}, w_{4}, y_{2}^{\prime}\right]$ contains a $K_{4}^{-}$.

If $w_{2} u_{2}, w_{1} u_{4} \in E(G)$, then by Claim 4 (b) for $j=3, y_{1}, y_{2}, y_{2}^{\prime}$ are not neighbors of $u_{3}$. By Claim 5 (b) for $h=4$, either $u_{1} y_{1}^{\prime} \notin E(G)$ or $e_{G}\left(u_{2}, Y_{2}\right)=0$. So, by (13), $u_{1} y_{1}^{\prime} \notin E(G)$. Now by Claim 5 (b) for $h=1$, either $u_{4} y_{1}^{\prime} \notin E(G)$, or $e_{G}\left(\left\{u_{2}, u_{3}\right\}, Y_{2}\right)=0$. So, by (13), $u_{4} y_{1}^{\prime} \notin E(G)$ and all other edges in $E(S, U)$ are present. Thus, $G\left[y_{1}^{\prime}, u_{2}, u_{3}, w_{1}\right]$ contains a $K_{4}^{-},\left\{y_{1}, w_{3}, w_{4}, y_{2}\right\}$ is a $Y$-connector, and $\left(u_{1}, y_{2}^{\prime}, w_{2}, u_{4}\right)$ is a path in $G$.

The last possibility is that $w_{1} u_{3}, w_{2} u_{1} \in E(G)$. In this case, by Claim 4 (b) for $j=2$, $y_{1}, y_{2}, y_{2}^{\prime}$ are not neighbors of $u_{2}$. By Claim 5 (a) for $h=1$, either $e_{G}\left(u_{4}, Y_{2}\right)=0$ or $e_{G}\left(y_{1}^{\prime},\left\{u_{2}, u_{3}\right\}\right)=0$. Since $e_{G}\left(y_{1}^{\prime},\left\{u_{2}, u_{3}\right\}\right)>0$ by $(13)$, we conclude that $e_{G}\left(u_{4}, Y_{2}\right)=0$. Now by Claim 5 (a) for $h=4$, either $e_{G}\left(u_{1}, Y_{2}\right)=0$ or $e_{G}\left(y_{1}^{\prime},\left\{u_{2}, u_{3}\right\}\right)=0$. Both cases contradict (13).

Case 1.2: $e_{G}\left(\left\{u_{2}, u_{3}\right\},\left\{w_{1}, w_{2}\right\}\right)=4$. If both, $e_{G}\left(u_{1},\left\{w_{1}, w_{2}\right\}\right)>0$ and $e_{G}\left(u_{4},\left\{w_{1}, w_{2}\right\}\right)>$ 0 , then by Claim $4, e_{G}\left(\left\{u_{2}, u_{3}\right\}, Y\right) \leq 2$. This contradicts (13). So, we may assume that $e_{G}\left(u_{4},\left\{w_{1}, w_{2}\right\}\right)=0$. Then under the conditions of Case $1, e_{G}\left(u_{1},\left\{w_{1}, w_{2}\right\}\right)>0$.

Case 1.2.1: $u_{1} w_{2} \in E(G)$. By Claim 4, $u_{2} y_{1}, u_{2} y_{2}, u_{2} y_{2}^{\prime} \notin E(G)$. By Claim 5, either $e_{G}\left(u_{4}, Y_{2}\right)=0$ or $e_{G}\left(y_{1}^{\prime},\left\{u_{2}, u_{3}\right\}\right)=0$. So, by (13), at most one other edge in $E(Y, U)$ is missing and this edge must either be $u_{1} w_{2}$ or be in $E\left(Y,\left\{u_{1}, u_{4}\right\}\right)$. If $y_{1} u_{4} \in E(G)$, then $\left\{y_{1}^{\prime}, w_{1}, u_{3}, y_{2}^{\prime}\right\}$ is a $Y$-connector, $G\left[\left\{w_{2}, w_{3}, w_{4}, y_{2}\right\}=K_{4}\right.$, and $\left(u_{1}, u_{2}, y_{1}, u_{4}\right)$ is a path in $G$. Otherwise $u_{1} w_{1} \in E(G)$ and hence $\left\{y_{1}, u_{2}, w_{2}, y_{2}\right\}$ is a $Y$-connector, $G\left[\left\{y_{1}^{\prime}, w_{3}, w_{4}, y_{2}^{\prime}\right\}\right]=K_{4}^{-}$, and $\left(u_{1}, w_{1}, u_{3}, u_{4}\right)$ is a path in $G$.

Case 1.2.2: $u_{1} w_{2} \notin E(G)$. Then $u_{1} w_{1} \in E(G)$. By Claim $4, u_{2} y_{1}^{\prime}, u_{2} y_{2}, u_{2} y_{2}^{\prime} \notin E(G)$. By Claim 5, either $u_{4} y_{1}^{\prime} \notin E(G)$ or $e_{G}\left(Y_{2},\left\{u_{2}, u_{3}\right\}\right)=0$. So, by $(13), u_{4} y_{1}^{\prime} \notin E(G)$ and at most one other edge in $E(Y, U)$ is missing. Moreover, the missing edge, if exists, must be in $E\left(Y,\left\{u_{1}, u_{4}\right\}\right)$. Then $u_{1}$ has a neighbor, say $z$, in $Y_{2}$. Therefore, $Y-y_{1}^{\prime}-z+w_{2}+w_{4}$ is a $Y$-connector, $G\left[\left\{\left\{y_{1}^{\prime}, w_{1}, w_{3}, u_{2}\right\}\right]\right.$ contains a $K_{4}^{-}$, and $\left(u_{1}, z, u_{3}, u_{4}\right)$ is a path in $G$.

Case 2: $d_{3}\left(u_{2},\left\{w_{1}, w_{2}\right\}\right)+d_{3}\left(u_{3},\left\{w_{1}, w_{2}\right\}\right) \leq 8$. Вy $(13), d_{3}\left(u_{2}, Y\right)+d_{3}\left(u_{3}, Y\right) \geq 17$. Ву symmetry, we may assume that $d_{3}\left(u_{2}, Y\right) \geq 9$. Then by Lemma 1 , we only need to consider the 6 configurations in Figure 1 with $i=2$.

Note that for each configuration in Figure 1, there exist $y \in Y_{1}, z \in Y_{2}$ adjacent to both $u_{1}$ and $u_{3}$. Let $y^{\prime} \in Y_{1}-y$ and $z^{\prime} \in Y_{2}-z$. Then in all cases, $y^{\prime} u_{2}, z u_{4} \in E(G)$.

If $w_{2} u_{2} \in E(G)$, then $\left\{y^{\prime}, u_{2}, w_{2}, z\right\}$ is a $Y$-connector, $G\left[\left\{w_{1}, w_{3}, w_{4}, z^{\prime}\right\}\right]$ contains a $K_{4}^{-}$, and $\left(u_{1}, y, u_{3}, u_{4}\right)$ is a path in $G$. Similarly, if $w_{1} u_{2} \in E(G)$, then $\left\{y_{1}, w_{3}, w_{2}, z^{\prime}\right\}$ is a $Y$ connector, $G\left[\left\{w_{1}, w_{3}, u_{2}, y_{1}^{\prime}\right\}\right]$ contains a $K_{4}^{-}$, and $\left(u_{1}, z, u_{3}, u_{4}\right)$ is a path in $G$. So,

$$
w_{2} u_{2} \notin E(G) \text { and } \quad w_{1} u_{2} \notin E(G) .
$$

Note that in all cases except $(\mathrm{E}), d_{3}\left(u_{2}, Y\right)+d_{3}\left(u_{3}, Y\right)=17$ and in $(\mathrm{E}), d_{3}\left(u_{2}, Y\right)+$ $d_{3}\left(u_{3}, Y\right)=18$. So, by $(13), d_{3}\left(u_{2},\left\{w_{1}, w_{2}\right\}\right)+d_{3}\left(u_{3},\left\{w_{1}, w_{2}\right\}\right)$ is at least 7 in Case (E) and at least 8 otherwise. Thus in the cases other than (E), we do not have other non-edges between $\left\{w_{1}, w_{2}\right\}$ and $\left\{u_{1}, u_{2}, u_{3}, u_{4}\right\}$. Therefore, $G\left[\left\{y, w_{1}, w_{3}, w_{4}\right\}\right]$ contains a $K_{4}^{-},\left\{y^{\prime}, u_{2}, u_{3}, z^{\prime}\right\}$ is a 
$Y$-connector, and $\left(u_{1}, z, w_{2}, u_{4}\right)$ is a path in $G$. This argument also works for $(\mathrm{E})$ if the missing edge is incident with $w_{1}$. If not, then $w_{1}$ is adjacent to both $u_{1}$ and $u_{4}$ and $y$ is adjacent to at least one of them. Thus, $\left\{y^{\prime}, u_{2}, u_{3}, z^{\prime}\right\}$ is a $Y$-connector, $G\left[\left\{z, w_{2}, w_{3}, w_{4}\right\}\right]=K_{4}$, and either $\left(u_{1}, y, w_{1}, u_{4}\right)$ or $\left(u_{1}, w_{1}, y, u_{4}\right)$ is a path in $G$.

Lemma 7. Let $F_{2}$ be a k-lasso-component of $H^{\prime}$ that consists of a path $\left(u_{1}, \ldots, u_{k}\right)$ and edges $u_{1} u_{3}$ and $u_{k-2} u_{k}$, where $k \geq 6$. Denote $T=\left\{u_{1}, u_{2}, u_{3}\right\}$. Suppose that $T \cap V\left(F_{1}\right)=\emptyset$ and $e_{G}\left(T,\left\{w_{1}, w_{2}\right\}\right)=6$. If $G$ does not contain an $H$-approximation slightly better than $H^{\prime}$, then (a) no vertex in $Y-y_{1}^{\prime}$ has a neighbor in $\left\{u_{1}, u_{2}\right\}$;

(b) if $u_{3}$ has a neighbor in $Y_{2}$, then $y_{1}^{\prime}$ has no neighbor in $\left\{u_{1}, u_{2}\right\}$;

(c) $e_{G}(Y, T) \leq 4$.

Proof. Suppose by contradiction that $e_{G}\left(Y_{2},\left\{u_{1}, u_{2}\right\}\right)>0$. By symmetry, we may assume that $y_{2} u_{1} \in E(G)$. Define $y=y_{1}$ if $y_{1} w_{1} \in E(G)$ and let $y=y_{1}^{\prime}$ otherwise. Let $y^{\prime} \in Y_{1}-y$. Then $\left\{y_{2}, u_{1}, w_{1}, y\right\}$ is a $Y$-connector, $G\left[\left\{y^{\prime}, w_{3}, w_{4}, y_{2}^{\prime}\right\}\right]$ contains a $K_{4}^{-}$, and $G\left[T-u_{1}+w_{2}\right]$ contains a $K_{3}$. The proof for $y_{1}$ in place of $y_{2}$ is a bit simpler. This proves (a).

Suppose now that $e_{G}\left(y_{1}^{\prime},\left\{u_{1}, u_{2}\right\}\right)>0$ and $e_{G}\left(u_{3}, Y_{2}\right)>0$. By symmetry, we may assume that $y_{1}^{\prime} u_{1} \in E(G)$ and $u_{3} y_{2} \in E(G)$. Then $\left\{y_{1}, w_{3}, w_{4}, y_{2}^{\prime}\right\}$ is a $Y$-connector, $G\left[\left\{y_{1}^{\prime}, w_{1}, u_{1}, u_{2}\right\}\right]$ contains a $K_{4}^{-}$, and $G\left[T-u_{1}-u_{2}+w_{2}+y_{2}\right]$ contains a $K_{3}$. This proves (b), and (c) follows from (a) and (b).

Now we return to the remaining cases of $D$ satisfying (12).

If $H^{\prime}[D]=C_{k}=\left(u_{1}, \ldots, u_{k}\right), k \geq 5$, is a component of $H^{\prime}$, then consider $\sum=\sum_{i=1}^{k}\left(d_{3}\left(u_{i}, S\right)+\right.$ $\left.d_{3}\left(u_{i+1}, S\right)\right)$, where indices count modulo $k$. Then $\sum=6 e_{G}(S, D)>24 k$, and so there exists $1 \leq i \leq k$ such that $d_{3}\left(u_{i}, S\right)+d_{3}\left(u_{i+1}, S\right)>24$, a contradiction to Lemma 6 .

Suppose now that $H^{\prime}[D]$ is a $D_{k}$-component of $H^{\prime}$ that contains a path $\left(u_{1}, \ldots, u_{k}\right)$ and the edges $u_{1} u_{3}$ and $u_{k-2} u_{k}$ for some $k \geq 6$. As in the proof of Lemma 3 , let $T_{1}=\left\{u_{1}, u_{2}, u_{3}\right\}$, $T_{2}=\left\{u_{k-2}, u_{k-1}, u_{k}\right\}$, and let $P$ denote the path $\left(u_{4}, \ldots, u_{k-3}\right)$. We may assume that $D \cup Y$ cannot be partitioned into a $Y$-connector and a set $W$ such that $G[W]$ contains $D_{k}$, since otherwise $G$ contains a subgraph slightly better than $H^{\prime}$. Thus Claim 1 and Claim 2 hold true.

If $k=6$, then by Claim $1, e_{G}\left(Y, T_{i}\right) \leq 8$ for $i=1,2$. Hence $e_{G}\left(\left\{w_{1}, w_{2}\right\}, D\right)>24-8-8=8$. By symmetry, we may assume that $e_{G}\left(w_{1}, T_{1}\right)=3$ and $e_{G}\left(w_{4}, T_{2}\right) \geq 2$. Then $G\left[T_{1}+w_{1}\right]=K_{4}$, $G\left[T_{2} \cup\left\{w_{2}, w_{3}, y_{2}\right\}\right]$ contains a $D_{6}$, and $Y_{1}+w_{4}+y_{2}^{\prime}$ is a $Y$-connector.

If $k=7$, then by Lemma $3, e_{G}(Y, D) \leq 18$, and so $e_{G}\left(\left\{w_{1}, w_{2}\right\}, D\right)>28-18=10$. If $u_{4} w_{2} \in E(G)$ and $w_{1}$ has at least two neighbors in $T_{i}$ for some $i=1,2$, then $G\left[T_{i}+w_{1}\right]$ contains a $K_{4}^{-}, G\left[T_{3-i} \cup\left\{u_{4}, w_{2}, w_{3}, y_{2}\right\}\right]$ contains a $D_{7}$, and $Y_{1}+w_{4}+y_{2}^{\prime}$ is a $Y$-connector. Thus if $u_{4} w_{2} \in E(G)$, then $e_{G}\left(w_{1}, D\right) \leq 3$ and hence $e_{G}\left(\left\{w_{1}, w_{2}\right\}, D\right) \leq 7+3=10$, a contradiction. A symmetric argument works for $w_{1}$ in place of $w_{2}$. So we may assume that $u_{4} w_{1}, u_{4} w_{2} \notin E(G)$. 
Then $e_{G}\left(T_{1} \cup T_{2},\left\{w_{1}, w_{2}\right\}\right) \geq 11$, and so for some $i \in\{1,2\}, e_{G}\left(T_{i},\left\{w_{1}, w_{4}\right\}\right)=6$. By Lemma $7, e_{G}\left(T_{i}, Y\right) \leq 4$. Then by Claim $1, e_{G}(Y, D) \leq 4+8+4=16$, which in turn gives that $e_{G}\left(T_{1} \cup T_{2},\left\{w_{1}, w_{2}\right\}\right) \geq 29-16=13$, an impossibility.

If $k=8$, then by Lemma $3, e_{G}(Y, D) \leq 21$, and so $e_{G}\left(\left\{w_{1}, w_{2}\right\}, D\right)>32-21=11$. If $e_{G}\left(\left\{w_{1}, w_{2}\right\},\left\{u_{4}, u_{5}\right\}\right)=0$, then $e_{G}\left(T_{1} \cup T_{2},\left\{w_{1}, w_{2}\right\}\right)=12$. Hence by Claim $7, e_{G}\left(Y, T_{1} \cup\right.$ $\left.T_{2}\right) \leq 8$, and so $e_{G}(Y, D) \leq 8+2|Y|=16$. Thus in this case, $e_{G}\left(\left\{w_{1}, w_{2}\right\}, D\right)>32-16=16$, an impossibility. If $w_{1} u_{4} \in E(G)$ and $w_{2}$ has at least two neighbors in $T_{1}$, then $G\left[T_{1}+w_{2}\right]$ contains a $K_{4}^{-}, Y-y_{1}^{\prime}+w_{4}$ is a $Y$-connector, and $G\left[T_{2} \cup\left\{u_{5}, u_{4}, y_{1}^{\prime}, w_{1}, w_{3}\right\}\right]$ contains a $D_{8}$. Repeating this argument with the switched roles of $w_{1}$ and $w_{2}$ and/or of $u_{4}$ and $u_{5}$, we conclude that if $e_{G}\left(\left\{w_{1}, w_{2}\right\},\left\{u_{4}, u_{5}\right\}\right)=j$, then $e_{G}\left(\left\{w_{1}, w_{2}\right\}, T_{1} \cup T_{2}\right) \leq 12-2 j$, and hence $e_{G}\left(\left\{w_{1}, w_{2}\right\}, D\right) \leq 12-j<12$, a contradiction.

Let $k \geq 9$. As in the proof of Lemma 3 , we consider

$$
\begin{gathered}
S_{1}^{\prime}=\sum_{i=1}^{3} d\left(u_{i}, S\right)+\frac{5}{6} d\left(u_{4}, S\right)+\frac{1}{2} d\left(u_{5}, S\right)+\frac{1}{6} d\left(u_{6}, S\right), \\
S_{2}^{\prime}=\frac{1}{6} d\left(u_{k-6}, S\right)+\frac{1}{2} d\left(u_{k-5}, S\right)+\frac{5}{6} d\left(u_{k-4}, S\right)+\sum_{i=k-3}^{k} d\left(u_{i}, S\right) . \\
\text { and } S^{\prime}=S_{1}^{\prime}+\frac{1}{6} \sum_{i=5}^{k-4}\left(d_{3}\left(u_{i}, S\right)+d_{3}\left(u_{i+1}, S\right)\right)+S_{2}^{\prime}
\end{gathered}
$$

Note that these sums are well defined for $k \geq 9$ and that $S^{\prime}=e_{G}(S, D) \geq 4 k+1$. By Lemma $6, d_{3}\left(u_{i}, S\right)+d_{3}\left(u_{i+1}, S\right) \leq 24$ for $5 \leq i \leq k-4$ when $k \geq 9$. Thus $S_{1}^{\prime}+S_{2}^{\prime} \geq 37$. We may assume that $S_{1}^{\prime} \geq 18.5$. By Claim $2, Y$ contributes at most 12 to $S_{1}^{\prime}$.

If $u_{4} w_{1} \in E(G)$ and $e_{G}\left(w_{2}, T_{1}\right) \geq 2$, then $G\left[T_{1}+w_{2}\right]$ contains a $K_{4}^{-},\left\{y_{1}, w_{4}, y_{2}, y_{2}^{\prime}\right\}$ is a $Y$-connector, and $G\left[D-T_{1}+\left\{y_{1}^{\prime}, w_{1}, w_{3}\right\}\right]$ contains a $D_{k}$. Similar statement holds with the switched roles of $w_{1}$ and $w_{2}$. So, if $e_{G}\left(\left\{w_{1}, w_{2}\right\}, u_{4}\right)=j$, then $e_{G}\left(\left\{w_{1}, w_{2}\right\}, T_{1}\right) \leq 6-2 j$, and hence $S_{1}^{\prime} \leq 12+(6-2 j)+j \cdot \frac{5}{6}+2 \cdot \frac{1}{2}+2 \cdot \frac{1}{6}$. For $j \geq 1$, this expression is less than 18.5 , so $j=0$. If $e_{G}\left(\left\{w_{1}, w_{2}\right\}, T_{1}\right) \leq 5$, then $S_{1}^{\prime} \leq 12+5+\frac{4}{3}<18.5$. Thus, $e_{G}\left(\left\{w_{1}, w_{2}\right\}, T_{1}\right)=6$ and by Lemma $7, e_{G}\left(Y, T_{1}\right) \leq 4$. Then

$$
S_{1}^{\prime} \leq 4+4\left(\frac{5}{6}+\frac{1}{2}+\frac{1}{6}\right)+6+2\left(\frac{1}{2}+\frac{1}{6}\right)<18
$$

a contradiction.

Thus all cases when $H^{\prime}[D]$ is a component of $H^{\prime}$ disjoint from $F_{1}$ are considered. Now, suppose that the $Y_{1}$-block and the $Y_{2}$-block is the same component $H^{\prime}[B]$ of $H^{\prime}$ and $D=$ $B-Y$. By the definition of a gadget and (10), $H^{\prime}[B]$ is a double lasso, say $\left(z_{1}, \ldots, z_{k}\right)$, where $z_{1}=y_{1}, z_{2}=y_{1}^{\prime}, z_{k-1}=y_{2}^{\prime}, z_{k}=y_{2}$. By $(11)$, since $e_{G}\left(S, V(G)-F_{1}\right) \geq 4 n-27=4(n-8)+5$ and for every component $F_{2}$ of $H^{\prime}$ distinct from $B, e_{G}\left(S, F_{2}\right) \leq 4\left|F_{2}\right|$, we have $e_{G}(S, B-Y) \geq$ $4(|B|-4)+5$. Since $e_{G}(S, B-Y) \leq|S||B-Y|=6|B-Y|$, we have $k \geq 7$. As in the proof of the Dirac's theorem, if for some $3 \leq i \leq k-4, z_{i} z_{k} \in E(G)$ and $z_{1} z_{i+1}$, then $G[B]$ contains 
Hamiltonian cycle $\left(z_{1}, z_{2}, \ldots, z_{i}, z_{k}, z_{k-1}, \ldots, z_{i+1}\right)$, a contradiction. Since $z_{1}$ and $z_{2}$ (and $z_{k}$ and $\left.z_{k-1}\right)$ are equivalent in $B$, we conclude that $e_{G}(Y, B-Y) \leq 2(1+(k-4))$. It follows that $e_{G}(S, B-Y) \leq 2(1+(k-4))+2(k-4)=2+4(k-4)$, a contradiction.

Finally, suppose that $B_{1} \neq B_{2}$. By (11), for some $j \in\{1,2\}$,

$$
e_{G}\left(S, B_{j}-Y_{j}\right) \geq 3+4\left(\left|B_{j}\right|-2\right) .
$$

Suppose that $H^{\prime}\left[B_{j}\right]$ is a double lasso $\left(z_{1}, \ldots, z_{k}\right)$, where $\left\{z_{1}, z_{2}\right\}=\left\{y_{j}, y_{j}^{\prime}\right\}$. Similarly to the previous paragraph, if for some $3 \leq i \leq k-4$ and some $z \in Y_{3-j}, z_{i} z \in E(G)$ and $z_{1} z_{i+1}$, then $G\left[B_{1} \cup B_{2}\right]$ contains a double lasso that contains $B_{3-j}$ and the path $\left(z, z_{i}, z_{i-1}, \ldots, z_{1}, z_{i+1}, z_{i+2}, \ldots, z_{k}\right)$. By the symmetry between $z_{2}$ and $z_{1}$, we conclude that $e_{G}\left(Y, B_{j}-Y_{j}\right) \leq 2(1+(k-2))$, a contradiction to (15). This proves the lemma.

\section{Proof of Lemma 5}

In this section, we prove Lemma 5 . Lemma 5 is a technical lemma which handles one complicated situations in Stage 3 and 4. Roughly speaking, it stated a half-gadget may be improved to a $Z$-attachment (which will give us $Y$-connector) under the given edge conditions.

Assume by contradiction that the lemma fails for some choice of $Z, T$, and $D$. Everywhere in this section we use notation $T=\left\{u_{1}, u_{2}, u_{3}\right\}$. Because of (4), it will be convenient to give to every edge in $E(Z, D)$ weight 1.5 and to every edge in $E(T, D)$ weight 1 . Accordingly, for every $A \subseteq D$ and $B \subseteq T \cup Z$, we define $w(A, B)=e_{G}(A, T \cap B)+1.5 e_{G}(A, Z \cap B)$ and $w_{\bar{G}}(A, B)=e_{\bar{G}}(A, T \cap B)+1.5 e_{\bar{G}}(A, Z \cap B)$. In these terms, (4) can be rewritten as $w(D, T \cup Z)>4|D|$, or, equivalently,

$$
w_{\bar{G}}(D, T \cup Z)<2|D| .
$$

If $D=\{x\}$, then by (4), $x$ has a neighbor $z_{i} \in Z$ and a neighbor in $T$. So, $D \cup T-z_{3-i}+x$ is a $Z$-attachment.

Suppose that $D=\left\{x_{1}, x_{2}\right\}$. By $(4), w(D, T \cup Z) \geq 8.5$. If there is a matching of size 2 connecting $D$ with $Z$ (say, with edges $z_{1} x_{1}$ and $z_{2} x_{2}$ ), then some vertex of $Z$ (say, $x_{1}$ ) still has a neighbor in $T$. In this case, $D \cup T-z_{2}+x_{1}$ is a $Z$-attachment and $G\left[\left\{x_{2}, z_{2}\right\}\right]=K_{2}$. Otherwise, at most two edges connect $Z$ with $D$ and hence all edges connecting $T$ with $D$ are present. Moreover, there is an edge connecting $D$ with $Z$, say, $x_{1} z_{1}$. Hence for any $c \in T$, $D \cup T-c+x_{1}$ is a $Z$-attachment and $G\left[\left\{x_{2}, c\right\}\right]=K_{2}$.

If $H^{\prime}[D]=K_{3}$, then $D$ has a neighbor in $Z$ and thus $G[D \cup Z]$ is a $Z$-attachment.

Let $D=\left\{x_{1}, x_{2}, x_{3}, x_{4}\right\}$ and $H^{\prime}[D]=K_{4}^{-}$with edge $x_{2} x_{4}$ missing. By (4),

$$
w(D, T \cup Z) \geq 16.5 \text {. }
$$

If some $x \in\left\{x_{2}, x_{4}\right\}$ has at least two neighbors in $T$, then some other vertex in $D$ still has a neighbor in $Z$, and hence $D \cup Z-x$ is a $Z$-attachment. Suppose now that $x_{2}$ has exactly one neighbor in $T$. If $x_{2}$ also has a neighbor $z \in Z$, and $z^{\prime} \in Z-z$, then $T+z+x_{2}$ is a $Z$-attachment, and to avoid $K_{4}^{-}$in $G\left[D-x_{2}+z^{\prime}\right], z^{\prime}$ has two non-neighbors in $D-x_{2}$. In this case, to satisfy (17), $x_{2} z^{\prime} \in E(G)$, but then, as above, $z$ has two non-neighbors in 
$D-x_{2}$, a contradiction to (17). So, $x_{2}$ has no neighbors in $Z$. But then, by (17), $x_{4}$ has a neighbor in $T$ and a neighbor in $Z$, a contradiction, as above. Thus our assumption is false and $e_{G}\left(T,\left\{x_{2}, x_{4}\right\}\right)=0$. It follows that at most one other edge between $D$ and $T \cup Z$ is missing. In particular, we may assume that $z_{1}$ is adjacent to all vertices in $D$ and that $z_{2} x_{3} \in E(G)$. Then $Z \cup T-z_{1}+x_{3}$ is a $Z$-attachment and $G\left[D-x_{3}+z_{1}\right]$ contains a $K_{4}^{-}$.

Let $H^{\prime}[D]$ be a 6-lasso with triangles $T_{1}$ and $T_{2}$. If $T$ has a neighbor in $T_{i}$ and $Z$ has a neighbor in $T_{3-i}$, then $Z \cup T_{3-i}$ is a $Z$-attachment, a contradiction. So $w(D, T \cup Z) \leq 18<$ $24=4|D|$, a contradiction to $(4)$.

The remaining three cases, when $H^{\prime}[D] \in\left\{C_{5}^{+}, D_{9}, D_{7}\right\}$, need much longer proofs.

Case 1: $H^{\prime}[D]=C_{5}^{+}$. Let $H^{\prime}[D]$ be a 5 -cycle $\left(y_{1}, y_{2}, y_{3}, y_{4}, y_{5}\right)$ with chord $y_{2} y_{5}$. By (4),

$$
w(D, T \cup Z) \geq 20.5 \text {. }
$$

We start from a sequence of short claims (T1-T9). These claims explain where the edges between $D$ and $T \cup Z$ could be.

Since we assumed that the lemma fails for $Z, T$, and $D$, the first statement follows.

(T1) If $T \cup D$ can be partitioned $T \cup D=W_{1} \cup W_{2}$ so that $G\left[W_{1}\right]=K_{3}$ and $G\left[W_{2}\right] \supseteq C_{5}^{+}$, then $e_{G}\left(Z, W_{1}\right)=0$.

(T2) If $e_{G}(D, T) \geq 6$, then for some $y_{i}, y_{j} \in D, G\left[T+y_{i}+y_{j}\right]$ contains $C_{5}^{+}$.

Proof of (T2): Suppose that (T2) fails. Every 5 -vertex graph with at least 8 edges contains $C_{5}^{+}$. So, if $e_{G}\left(y_{i}, T\right)=3$ for some $y_{i} \in D$, then $e_{G}\left(y_{i-1}, T\right)=0, e_{G}\left(y_{i+1}, T\right)=0$, and $e_{G}\left(y_{j}, T\right) \leq 1$ for $j=i-2, i+2$. This yields $e_{G}(D, T) \leq 5$, a contradiction. So, $e_{G}\left(y_{i}, T\right) \leq 2$ for all $y_{i} \in D$. Then there are two adjacent $y_{i}$ and $y_{j}$ such that $e_{G}\left(y_{i}, T\right)=2$ and $e_{G}\left(y_{j}, T\right) \geq$ 1. For these $i$ and $j, G\left[T+y_{i}+y_{j}\right]$ contains $C_{5}^{+}$.

(T3) $e_{G}(Z, D) \leq 8$ and $e_{G}(T, D) \geq 9$.

Proof of (T3): By $(18), e_{G}(D, T)>20-1.5 e_{G}(D, Z) \geq 5.5$. So $e_{G}(T, D) \geq 6$, with equality only if $e_{G}(D, Z)=10$. Suppose that $e_{G}(D, Z)=10$. In this case, for any 3 -vertex subset $W$ of $D, G[W]$ has an edge, and hence the set $Z \cup W$ is a $Z$-attachment. Furthermore, by (T2) for some two vertices $y_{i}, y_{j} \in D, G\left[T+y_{i}+y_{j}\right]$ contains $C_{5}^{+}$. So, the lemma holds in this case. Thus, $e_{G}(D, Z) \leq 9$ and hence $e_{G}(T, D) \geq 7$.

Suppose $e_{G}(D, Z)=9$. Then for any $i \in\{1, \ldots, 5\}, Z \cup\left\{v_{i-1}, v_{i}, v_{i+1}\right\}$ is a $Z$-attachment. Since $e_{G}\left(T, D^{\prime}\right) \geq 7$, for some $j \in\{1, \ldots, 5\}, G\left[T+y_{j}+j_{j+1}\right]$ contains $C_{5}^{+}$. This contradiction shows that $e_{G}(Z, D) \leq 8$ and hence $e_{G}(D, T)>20-1.5 e_{G}(D, Z) \geq 8$.

(T4) $E\left(T,\left\{y_{3}, y_{4}\right\}\right)$ does not contain a matching of size two. As a result, $e_{G}\left(T,\left\{y_{3}, y_{4}\right\}\right) \leq$ 3 .

Proof of (T4): Otherwise, $T \cup\left\{y_{3}, y_{4}\right\}$ contains a $C_{5}^{+}$. By (T1), there is no edge between $Z$ and the triangle $\left(y_{1}, y_{2}, y_{5}\right)$. Then $e_{G}(Z, D) \leq 4$ and so by $(18), e_{G}(T, D) \geq 15$. On the other hand, by (18), $1.5 e_{G}(Z, D)>20-|T||D|=5$, and hence $e_{G}(Z, D) \geq 4$. This means that all edges between $Z$ and $\left\{y_{3}, y_{4}\right\}$ and between $T$ and $D$ are present. So for any $u \in T$, $G\left[D-y_{3}+u\right]$ contains a $C_{5}^{+}$and $Z \cup T-u+y_{3}$ is a $Z$-attachment. 
(T5) $T \cup D$ has no partition into $T^{\prime}$ and $D^{\prime}$ such that $G\left[T^{\prime}\right]=K_{3}$ and $G\left[D^{\prime}\right] \supset C_{5}^{+}$with $\left|T \cap T^{\prime}\right| \leq 1$.

Proof of (T5): If there is such a partition $T \cup D=T^{\prime} \cup D^{\prime}$, then by (T1), $e_{G}\left(Z, T^{\prime}\right)=0$. It follows that $e_{G}(Z, D) \leq 6$, and so $e_{G}(T, D) \geq 12$. Since in this case $e_{G}\left(T,\left\{y_{3}, y_{4}\right\}\right) \geq 3$, by (T4), we may assume that $e_{G}\left(y_{3}, T\right)=0$. Hence $e_{G}\left(T, D-y_{3}\right)=12$. If $e_{G}\left(Z,\left\{y_{1}, y_{5}\right\}\right)>0$, then for any $u \in T, G\left[T-u+y_{2}+y_{3}+y_{4}\right]$ contains a $C_{5}^{+}$, and $Z+u+y_{1}+y_{5}$ is a $Z$-attachment, a contradiction. So $e_{G}\left(Z,\left\{y_{1}, y_{5}\right\}\right)=0$, and hence all edges connecting $Z$ and $\left\{y_{2}, y_{3}, y_{4}\right\}$ are present. Now $Z+y_{2}+y_{3}+y_{4}$ is a $Z$-attachment, and $G\left[T+y_{1}+y_{5}\right]$ contains a $C_{5}^{+}$, a contradiction.

(T6) If $e_{G}\left(T,\left\{y_{1}, y_{2}, y_{5}\right\}\right) \geq 8$, then $e_{G}\left(T,\left\{y_{3}, y_{4}\right\}\right)=0$.

Proof of (T6): Assume by contradiction that $e_{G}\left(T,\left\{y_{1}, y_{2}, y_{5}\right\}\right) \geq 8$ and that $u_{1} y_{4} \in E(G)$. Since at most one edge in $E\left(T,\left\{y_{1}, y_{2}, y_{5}\right\}\right)$ is missing and we can switch the roles of $u_{2}$ and $u_{3}$, we may assume that $u_{3} y_{1}, u_{3} y_{5}, u_{2} y_{2} \in E(G)$. So, if $u_{1} y_{2} \in E(G)$, then $G\left[\left\{u_{3}, y_{1}, y_{5}\right\}\right]=K_{3}$, and $G\left[\left\{u_{1}, u_{2}, y_{2}, y_{3}, y_{4}\right\}\right]$ contains a $C_{5}^{+}$, a contradiction to (T5). Thus $u_{1} y_{2} \notin E(G)$ and all other edges connecting $T$ with $\left\{y_{1}, y_{2}, y_{5}\right\}$ are present. Therefore, if $y_{4} u_{j} \in E(G)$ for some $j \in\{2,3\}$, then we switch the roles of $u_{1}$ and $u_{j}$ and the previous argument works. So, $e_{G}\left(y_{4}, T\right)=1$. Furthermore, if $y_{3}$ has a neighbor $u_{i} \in T$, then we can switch the roles of $y_{3}$ and $y_{4}$ and the roles of $y_{2}$ and $y_{5}$ : since $e_{G}\left(v_{5}, T\right)=3$, our argument works. Thus the last possibility is that $E\left(T,\left\{y_{3}, y_{4}\right\}\right)=\left\{y_{4} u_{1}\right\}$. Then $e_{G}(T, D)=9$ and so $e_{G}(Z, T) \geq 8$. On the other hand, since $G\left[\left\{y_{1}, u_{2}, u_{3}\right\}\right]=K_{3}$ and $G\left[D-y_{1}+u_{1}\right]$ contains a $C_{5}^{+}$, by (T1), $e_{G}\left(y_{1}, Z\right)=0$. It follows that $e_{G}\left(Z, D-y_{1}\right)=8$, and hence $T+y_{4}+z_{1}$ is a $Z$-attachment, and $G\left[D-y_{4}+z_{2}\right]$ contains a $C_{5}^{+}$.

(T7) $e_{G}(T, D) \leq 10$ and $e_{G}(Z, D) \geq 7$.

Proof of (T7): By symmetry, assume that $e_{G}\left(y_{4}, T\right) \geq e_{G}\left(y_{3}, T\right)$. If $e_{G}(T, D) \geq 11$, then by $(\mathrm{T} 4), e_{G}\left(T,\left\{y_{1}, y_{2}, y_{5}\right\}\right) \geq 8$, and so by $(\mathrm{T} 6), e_{G}\left(T,\left\{y_{3}, y_{4}\right\}\right)=0$, a contradiction to $e_{G}(T, D) \geq 11$.

(T8) $6 \leq e_{G}\left(T,\left\{y_{1}, y_{2}, y_{5}\right\}\right) \leq 7$.

Proof of (T8): By (T3) and (T4), $e_{G}\left(T,\left\{y_{1}, y_{2}, y_{5}\right\}\right) \geq 6$. Suppose that $e_{G}\left(T,\left\{y_{1}, y_{2}, y_{5}\right\}\right) \geq$ 8. Then by $(\mathrm{T} 6), e_{G}\left(T,\left\{y_{3}, y_{4}\right\}\right)=0$. By (T3), $e_{G}\left(T,\left\{y_{1}, y_{2}, y_{5}\right\}\right)=9$ and $e_{G}(Z, D)=8$. Since $G\left[T-u_{1}+y_{1}\right]=K_{3}$ and $G\left[D-y_{1}+u_{1}\right]$ contains a $C_{5}^{+}$, (T1) implies that $e_{G}\left(Z, D-y_{1}\right)=$ 8, and hence $Z \cup\left\{y_{2}, y_{3}, y_{4}\right\}$ is a $Z$-attachment, and $G\left[T+y_{1}+y_{5}\right]=K_{5}$, a contradiction.

(T9) $e_{G}\left(T,\left\{y_{3}, y_{4}\right\}\right) \leq 2$. So $e_{G}(Z, D)=8, e_{G}\left(T,\left\{y_{3}, y_{4}\right\}\right)=2$, and $e_{G}\left(T,\left\{y_{1}, y_{2}, y_{5}\right\}\right)=7$.

Proof of (T9): If $e_{G}\left(T,\left\{y_{3}, y_{4}\right\}\right) \geq 3$, then by (T4), $e_{G}\left(T,\left\{y_{3}, y_{4}\right\}\right)=3$ and exactly one of $y_{3}$ and $y_{4}$ (we may assume $y_{3}$ ) is adjacent to all vertices of $T$. Suppose first that $y_{5}$ has no neighbors in $T$. Then by (T8), $e_{G}\left(\left\{y_{1}, y_{2}\right\}, T\right)=6$ and hence $G\left[T+y_{1}-u_{1}\right]=K_{3}$ and $G\left[D-y_{1}+u_{1}\right]$ contains a $C_{5}^{+}$. By $(\mathrm{T} 0)$, this yields that $e_{G}\left(y_{1}, Z\right)=0$. On the other hand, since $e_{G}(T, D)=9$, by $(18)$, we have $e_{G}(Z, D) \geq 8$, and so $e_{G}\left(Z, D-y_{1}\right)=8$. In this case, $Z \cup\left\{y_{5}, y_{3}, y_{4}\right\}$ is a $Z$-attachment, and $G\left[T+y_{1}+y_{2}\right]=K_{5}$, a contradiction. 
So by symmetry we may assume that $u_{1} y_{5} \in E(G)$. Then for $j=2,3, G\left[\left\{y_{3}, y_{4}, y_{5}+u_{1}+\right.\right.$ $\left.\left.u_{j}\right\}\right]$ contains a $C_{5}^{+}$, and hence by (T5), $G\left[\left\{u_{2}, y_{1}, y_{2}\right\}\right]$ and $G\left[\left\{u_{3}, y_{1}, y_{2}\right\}\right]$ are not triangles. Thus $e_{G}\left(T-u_{1},\left\{y_{1}, y_{2}\right\}\right) \leq 2$. So by $(\mathrm{T} 8), e_{G}\left(y_{5}, T\right) \geq 2$, and we may assume that $u_{2} y_{5} \in$ $E(G)$. Repeating the argument with $u_{2}$ in place of $u_{1}$, we conclude that $e_{G}\left(u_{1},\left\{y_{1}, y_{2}\right\}\right) \leq 1$. So, by $(\mathrm{T} 8), e_{G}\left(y_{5}, T\right)=3$. It follows that $G\left[\left\{u_{1}, y_{1}, y_{2}\right\}\right]=K_{3}$ and $G\left[\left\{y_{3}, y_{4}, y_{5}, u_{2}, u_{3}\right\}\right]$ contains a $C_{5}^{+}$, a contradiction to (T5).

We now are ready to finish Case 1 . If for some $1 \leq i \leq 3, e_{G}\left(u_{i},\left\{y_{3}, y_{4}\right\}\right)=2$, then $G\left[\left\{u_{i}, y_{3}, y_{4}\right\}\right]=K_{3}$ and $G\left[\left\{y_{1}, y_{2}, y_{5}, u_{3}\right\} \cup\left(T-u_{i}\right)\right]$ contains a $C_{5}^{+}$, a contradiction to (T5). Thus by (T9) and (T4), we may assume that $y_{3} u_{1}, y_{3} u_{2} \in E(G)$. If $y_{5} u_{1}, y_{5} u_{2} \notin E(G)$, then by (T9), all other edges connecting $T$ and $\left\{y_{1}, y_{2}, y_{5}\right\}$ are present. Hence $G\left[\left\{u_{2}, u_{3}, y_{1}\right\}\right]=$ $K_{3}$ and $G\left[D-y_{1}+u_{1}\right]$ contains a $C_{5}^{+}$. So by $(\mathrm{T} 0), e_{G}\left(y_{1}, Z\right)=0$ and hence by (T9), $e_{G}\left(Z, D-y_{1}\right)=8$. It follows that $G\left[Z+y_{3}+y_{4}+y_{5}\right]$ is a $Z$-attachment, and $G\left[T+y_{1}+y_{2}\right]$ contains a $C_{5}^{+}$, a contradiction. Thus assume $u_{1} y_{5} \in E(G)$.

Now $G\left[u_{1}, u_{2}, y_{3}, y_{4}, y_{5}\right]$ contains a $C_{5}^{+}$. Then by (T5), $G\left[\left\{u_{3}, y_{1}, y_{2}\right\}\right] \neq K_{3}$, and hence for some $i \in\{1,2\}, u_{3} y_{i} \notin E(G)$.

Since $u_{3} y_{i} \notin E(G)$, by (T9), at most one edge is missing in $E\left(T-u_{3},\left\{y_{1}, y_{2}, y_{5}\right\}\right)$. So, by the symmetry between $u_{1}$ and $u_{2}$, we may assume that $u_{1} y_{1}, u_{1} y_{2}, u_{2} y_{5} \in E(G)$. Then $G\left[\left\{u_{1}, y_{1}, y_{2}\right\}\right]=K_{3}$ and $G\left[u_{2}, u_{3}, y_{3}, y_{4}, y_{5}\right]$ contains a $C_{5}^{+}$, a contradiction to (T5). This finishes Case 1.

Case 2: $H^{\prime}[D]=D_{9}$. Assume that $H^{\prime}[D]$ contains a path $\left(x_{1}, \ldots, x_{9}\right)$ and edges $x_{1} x_{3}$ and $x_{7} x_{9}$. Let $T_{1}=\left\{x_{1}, x_{2}, x_{3}\right\}$ and $T_{2}=\left\{x_{7}, x_{8}, x_{9}\right\}$. By (16),

$$
w_{\bar{G}}(D, T \cup Z)<18 .
$$

Claim 6. For $i=1,2, w_{\bar{G}}\left(T_{i}, Z \cup T\right) \geq 3$.

Proof. Suppose that $w_{\bar{G}}\left(T_{1}, Z \cup T\right) \leq 2.5$. Then $e_{G}\left(Z, T_{1}\right) \geq 5$ and $e_{G}\left(T_{1}, T\right) \geq 7$. Thus by symmetry we may assume that $e_{G}\left(z_{1}, T_{1}\right)=3$ and $e_{G}\left(z_{2}, T_{1}\right) \geq 2$. Let $x \in\left\{x_{1}, x_{2}\right\}$ be a neighbor of $z_{2}$. Since $e_{G}\left(T_{i}, T\right) \geq 7, x$ has a neighbor in $T$. Then $T+z_{2}+x$ is a $Z$-attachment, and $G\left[D-x+z_{1}\right]$ contains a $D_{9}$, a contradiction.

Claim 7. If $e_{G}\left(Z, T_{1}\right)>0$ (respectively, $e_{G}\left(Z, T_{2}\right)>0$ ), then $e_{G}\left(x_{4}, T\right)=0$ (respectively, $\left.e_{G}\left(x_{6}, T\right)=0\right)$.

Proof. If $e_{G}\left(Z, T_{1}\right)>0$ and $e_{G}\left(x_{4}, T\right)>0$, then $G\left[D-T_{1}+T\right]$ contains a $D_{9}$ and $Z \cup T_{1}$ is a $Z$-attachment.

Assume first that $e_{G}\left(Z, T_{2}\right)=0$, i.e., $w_{\bar{G}}\left(Z, T_{2}\right)=9$. Then by $(19), w_{\bar{G}}\left(Z, T_{1}\right)<18-9=9$, i.e., $e_{G}\left(Z, T_{1}\right)>0$. So by Claim $7, e_{G}\left(x_{4}, T\right)=0$, and hence by Claim 6 ,

$$
w_{\bar{G}}(D, T \cup Z)-w_{\bar{G}}\left(Z, T_{2}\right)-w_{\bar{G}}\left(T_{1}, Z \cup T\right)-w_{\bar{G}}\left(x_{4}, T\right)<18-9-3-3=3 .
$$


In particular, $e_{G}\left(x_{5}, T\right)>0$ and $e_{G}\left(\left\{x_{4}, x_{5}, x_{6}\right\}, Z\right) \geq 8$. Since at most one edge is missing in $E\left(\left\{x_{4}, x_{5}, x_{6}\right\}, Z\right)$, by the symmetry between $z_{1}$ and $z_{2}$, we may assume that $x_{5} z_{1}, x_{4} z_{2}, x_{6} z_{2} \in$ $E(G)$. Then $G\left[D-x_{5}+z_{2}\right]$ contains a $D_{9}$ and $T+z_{j}+x_{2}$ is a $Z$-attachment, a contradiction.

So we have $e_{G}\left(Z, T_{i}\right)>0$ for $i=1,2$. By Claim $7, x_{4}$ and $x_{6}$ have no neighbors in $T$.

Claim 8. $w_{\bar{G}}\left(T_{1} \cup T_{2}, Z \cup T\right) \geq 9$.

Proof. Suppose that

$$
w_{\bar{G}}\left(T_{1} \cup T_{2}, Z \cup T\right)<9 .
$$

Assume first that $e_{G}\left(Z, T_{1}\right) \geq 5$. Then for $i=1,2$, there exists $j=j(i) \in\{1,2\}$ such that $x_{i} z_{j} \in E(G)$ and $G\left[T_{1}-x_{i}+z_{3-j}\right]=K_{3}$. So for $i=1,2$, if $x_{i}$ has a neighbor in $T$, then $T+z_{j}+x$ is a $Z$-attachment, and $G\left[D-x_{i}+z_{3-j}\right]$ contains a $D_{9}$, a contradiction. Therefore, $e_{G}\left(\left\{x_{1}, x_{2}\right\}, T\right)=0$. Hence by $(20), w_{\bar{G}}\left(T_{2}, Z \cup T\right)<3$, a contradiction to Claim 6 . So, by the symmetry between $T_{1}$ and $T_{2}$, we conclude that $e_{G}\left(Z, T_{i}\right) \leq 4$ for $i=1,2$.

Since $e_{\bar{G}}\left(T_{1} \cup T_{2}, Z\right) \geq 4$, by $(20)$ we have $w_{\bar{G}}\left(T_{1} \cup T_{2}, T\right)<3$. Thus by the symmetry between $T_{1}$ and $T_{2}$, we may assume that $e_{G}\left(T, T_{1}\right) \geq 8$. If for some $i \in\{1,2\}, e_{G}\left(x_{i}, Z\right)>0$, then we can choose some $u \in T$ such that $G\left[T-u+x_{i}\right]=K_{3}$ and $G\left[T_{1}+u-x_{i}\right]=K_{3}$. In this case, $Z \cup(T-u)+x_{i}$ is a $Z$-attachment, and $G\left[D-x_{i}+u\right]$ contains a $D_{9}$. We conclude that $e_{G}\left(Z,\left\{x_{1}, x_{2}\right\}\right)=0$ and hence $w_{\bar{G}}\left(T_{1}, Z\right) \geq 6$. This together with (20) contradicts Claim 6 .

Claim 8 implies that

$$
w_{\bar{G}}\left(\left\{x_{4}, x_{5}, x_{6}\right\}, T \cup Z\right)-w_{\bar{G}}\left(\left\{x_{4}, x_{6}\right\}, T\right)<18-9-6=3 .
$$

So $e_{G}\left(x_{5}, T\right)>0$ and $e_{G}\left(\left\{x_{4}, x_{5}, x_{6}\right\}, Z\right) \geq 5$. Thus we may assume that $e_{G}\left(z_{1},\left\{x_{4}, x_{5}, x_{6}\right\}\right)=$ 3 and $e_{G}\left(z_{2},\left\{x_{4}, x_{5}, x_{6}\right\}\right) \geq 2$. By symmetry we may assume that $x_{4} z_{2} \in E(G)$. Then $T_{1}+x_{4}+z_{2}$ is a $Z$-attachment, and $G\left[T_{2} \cup T \cup\left\{x_{5}, x_{6}, z_{1}\right\}\right]$ contains a $D_{9}$, a contradiction.

Case 3: $H^{\prime}[D]=D_{7}$. Assume that $H^{\prime}[D]$ contains a path $\left(x_{1}, \ldots, x_{7}\right)$ and edges $x_{1} x_{3}$ and $x_{5} x_{7}$. Let $T_{1}=\left\{x_{1}, x_{2}, x_{3}\right\}$ and $T_{2}=\left\{x_{5}, x_{6}, x_{7}\right\}$. By (16),

$$
w_{\bar{G}}(D, T \cup Z)<14 \text {. }
$$

If $e_{G}\left(x_{4}, T\right)>0$ and $e_{G}\left(Z, T_{1}\right)>0$, then $G\left[D-T_{1}+T\right]$ contains a $D_{7}$ and $Z \cup T_{1}$ is a $Z$-attachment. So by symmetry, if $e_{G}\left(x_{4}, T\right)>0$, then $e_{G}\left(Z, T_{1} \cup T_{2}\right)=0$, and hence $w_{\bar{G}}(D, T \cup Z) \geq 1.5 \cdot 12=18$, a contradiction to $(21)$. So,

$$
e_{G}\left(x_{4}, T\right)=0 .
$$

Claim 9. For $i=1,2, w_{\bar{G}}\left(T_{i}, T \cup Z\right) \geq 5$, and if $w_{\bar{G}}\left(T_{i}, T \cup Z\right)=5$, then $e_{G}\left(Z, T_{i}\right)=4$ and $e_{G}\left(T, T_{i}\right)=7$. 
Proof. Assume that $w_{\bar{G}}\left(T_{1}, T \cup Z\right) \leq 5$. Then $e_{G}\left(Z,\left\{x_{1}, x_{2}\right\}\right)>0$, that is, for some $j \in\{1,2\}$, $x_{j}$ has a neighbor in $Z$. If $e_{G}\left(T_{1}, T\right) \geq 8$, then since at most one edge in $E\left(T_{1}, T\right)$ is missing, we may assume that $x_{j} u_{1}, x_{j} u_{2}, x_{3-j} u_{3}, x_{3} u_{3} \in E(G)$. In this case, $G\left[D-x_{j}+u_{3}\right]$ contains a $D_{7}$, and $Z \cup T-u_{3}+x_{j}$ is a $Z$-attachment. So, $e_{G}\left(T_{1}, T\right) \leq 7$, i.e.,

$$
w_{\bar{G}}\left(T_{1}, T\right) \geq 2 \text {. }
$$

Suppose now that $e_{G}\left(T_{1}, Z\right) \geq 5$. Since $w_{\bar{G}}\left(T_{1}, T\right) \leq 5$, for some $j \in\{1,2\}, x_{j}$ has a neighbor in $T$. Since at most one edge is missing in $E\left(T_{1}, Z\right)$, we may assume that $x_{j} z_{1}, x_{3-j} z_{2}, x_{3} z_{2} \in E(G)$. Then $G\left[D-x_{j}+z_{2}\right]$ contains a $D_{7}$, and $T+z_{1}+x_{j}$ is a $Z$ attachment. Thus $e_{G}\left(T_{1}, Z\right) \leq 4$, i.e., $w_{\bar{G}}\left(T_{1}, Z\right) \geq 3$. This together with (23) yields the claim.

By $(21)$ and $(22), w_{\bar{G}}\left(T_{1}, T \cup Z\right)+w_{\bar{G}}\left(T_{2}, T \cup Z\right) \leq 11$. So by Claim 9 , we may assume that $w_{\bar{G}}\left(T_{1}, T \cup Z\right)=5$, and therefore $e_{G}\left(Z, T_{1}\right)=4$ and $e_{G}\left(T, T_{1}\right)=7$. Also by (21), (22), and Claim 9,

$$
w_{\bar{G}}\left(x_{4}, Z\right)<14-w_{\bar{G}}(x, T)-w_{\bar{G}}\left(T_{1} \cup T_{2}, T \cup Z\right) \leq 14-3-10=1,
$$

which means that $x_{4} z_{1}, x_{4} z_{2} \in E(G)$. Since $e_{G}\left(Z, T_{1}\right)=4$, either $z_{1}$ or $z_{2}$ (say $z_{1}$ by symmetry) has at least two neighbors in $T_{1}$. Since $e_{G}\left(T, T_{1}\right)=7$, every vertex in $T_{1}$ has a neighbor in $T$. Then $T_{2}+x_{4}+z_{2}$ is a $Z$-attachment, and $G\left[T \cup T_{1}+z_{1}\right]$ contains a $D_{7}$, a contradiction.

\section{REFERENCES}

[1] M. Aigner and S. Brandt, Embedding Arbitrary Graphs of Maximum Degree Two, J. London Math. Soc. (2), 28 (1993) 39-51.

[2] N. Alon and E. Fischer, 2-factors in dense graphs, Discrete Math., 152 (1996), 13-23.

[3] B. Bollobás and S.E. Eldridge, Packing of graphs and applications to computational complexity, J. Comb. Theory Ser. B, 25 (1978), 105-124.

[4] P. A. Catlin, Subgraphs of graphs. I. Discrete Math., 10 (1974), 225-233.

[5] P. A. Catlin, Embedding subgraphs and coloring graphs under extremal degree conditions, Ph.D. Thesis, Ohio State Univ., Columbus, (1976).

[6] K. Corrádi and A. Hajnal, On the maximum number of independent circuits in a graph. Acta Math. Acad. Sci. Hung., 14 (1963), 423-439.

[7] B. Csaba, A. Shokoufandeh, and E. Szemerédi, Proof of a conjecture of Bollobás and Eldridge for graphs of maximum degree three, Combinatorica, 23 (2003), 35-72.

[8] G. Dirac, Some theorems on abstract graphs, Proc. London Math. Soc., 2 (1952), 69-81.

[9] H. Enomoto, On the existence of disjoint cycles in a graph, Combinatorica, 18 (1998), 487-492.

[10] G. Fan and H. A. Kierstead, Hamiltonian square-paths, J. Combin. Theory Ser. B 67 (1996), 167-182.

[11] P. Justesen, On independent circuits in finite graphs and a conjecture of Erdós and Posa, Annals of Discrete Math., 41 (1989), 299-306.

[12] H. A. Kierstead and A. V. Kostochka, Ore-type versions of Brooks' theorem, submitted.

[13] A. V. Kostochka and G. Yu, Ore-type graph packing problems. Combin. Probab. Comput. 16 (2007), no. $1,167-169$. 
[14] A. V. Kostochka and G. Yu, An Ore-type analogue of the Sauer-Spencer Theorem Graphs and Comb. 23 (2007) no. 4, 419-424.

[15] A. V. Kostochka and G. Yu, Ore-type degree conditions for the existence of 2-factors with short cycles, to appear in Disc. Math..

[16] O. Ore, Note on hamilton circuits, Amer. Math. Monthly, 67 (1960), 55.

[17] N. Sauer and J. Spencer, Edge disjoint placement of graphs, J. Combin. Theory Ser. B, 25 (1978), $295-302$.

[18] H. Wang, On the maximum number of independent cycles in a graph, Discrete Math., 205 (1999) $183-190$.

Alexandr V. KostochKa

Department of Mathematics, University of Illinois, Urbana, IL 61801, USA and Sobolev InstiTUte of Mathematics, Novosibirsk 630090, Russia.

E-mail address: kostochk@math.uiuc.edu

GEXIN YU

Department of Mathematics, College of William and Mary, Williamsburg, VA 23185, USA.

E-mail address: gyu@wm.edu 